\title{
BMJ Open Pre-admission interventions (prehabilitation) to improve outcome after major elective surgery: a systematic review and meta-analysis
}

Rachel Perry (10 , ${ }^{1}$ Georgia Herbert, ${ }^{1}$ Charlotte Atkinson, ${ }^{1}$ Clare England, ${ }^{1,2}$ Kate Northstone, ${ }^{3}$ Sarah Baos, ${ }^{4}$ Tim Brush, ${ }^{4}$ Amanda Chong, ${ }^{1}$ Andy Ness, ${ }^{1,5}$ Jessica Harris, ${ }^{4}$ Anne Haase, ${ }^{6}$ Sanjoy Shah, ${ }^{7}$ Maria Pufulete ${ }^{4}$

To cite: Perry R, Herbert G, Atkinson C, et al. Preadmission interventions (prehabilitation) to improve outcome after major elective surgery: a systematic review and meta-analysis. BMJ Open 2021;11:e050806. doi:10.1136/ bmjopen-2021-050806

- Prepublication history and additional supplemental material for this paper are available online. To view these files, please visit the journal online (http://dx.doi.org/10.1136/ bmjopen-2021-050806).

Received 02 March 2021 Accepted 06 July 2021

Check for updates

(C) Author(s) (or their employer(s)) 2021. Re-use permitted under CC BY. Published by BMJ.

For numbered affiliations see end of article.

Correspondence to

Dr Maria Pufulete;

maria.pufulete@bristol.ac.uk

\section{ABSTRACT}

Objective To determine the benefits and harms of preadmission interventions (prehabilitation) on postoperative outcomes in patients undergoing major elective surgery.

Design Systematic review and meta-analysis of randomised controlled trials (RCTs) (published or unpublished). We searched Medline, Embase, CENTRAL, DARE, HTA and NHS EED, The Cochrane Library, CINAHL, PsychINF0 and ISI Web of Science (June 2020).

Setting Secondary care.

Participants Patients ( $\geq 18$ years) undergoing major elective surgery (curative or palliative).

Interventions Any intervention administered in the preoperative period with the aim of improving postoperative outcomes.

Outcomes and measures Primary outcomes were 30-day mortality, hospital length of stay (LOS) and postoperative complications. Secondary outcomes included LoS in intensive care unit or high dependency unit, perioperative morbidity, hospital readmission, postoperative pain, heath-related quality of life, outcomes specific to the intervention, intervention-specific adverse events and resource use.

Review methods Two authors independently extracted data from eligible RCTs and assessed risk of bias and the certainty of evidence using Grading of Recommendations, Assessment, Development and Evaluation. Random-effects meta-analyses were used to pool data across trials.

Results 178 RCTs including eight types of intervention were included. Inspiratory muscle training (IMT), immunonutrition and multimodal interventions reduced hospital LoS (mean difference vs usual care: -1.81 days, $95 \% \mathrm{Cl}-2.31$ to $-1.31 ;-2.11$ days, $95 \% \mathrm{Cl}-3.07$ to $-1.15 ;-1.67$ days, $95 \% \mathrm{Cl}-2.31$ to -1.03 , respectively). Immunonutrition reduced infective complications (risk ratio (RR) $0.6495 \% \mathrm{Cl} 0.40$ to 1.01) and IMT, and exercise reduced postoperative pulmonary complications (RR 0.55, $95 \% \mathrm{Cl} 0.38$ to 0.80 , and RR $0.54,95 \% \mathrm{Cl} 0.39$ to 0.75 , respectively). Smoking cessation interventions reduced wound infections (RR $0.28,95 \% \mathrm{Cl} 0.12$ to 0.64 ). Conclusions Some prehabilitation interventions may reduce postoperative LoS and complications but the quality of the evidence was low.

PROSPERO registration number CRD42015019191.
Strengths and limitations of this study

- Unlike previous systematic reviews that focused on single interventions in single surgical populations, this review provides a summary of all types of prehabilitation interventions across all surgical populations.

- Comprehensive methods, with inclusion of published literature in all languages, alongside grey literature searching, to avoid publication bias.

- The large number of meta-analyses performed for related outcomes with data from the same individuals may lead to effect size multiplicity.

\section{INTRODUCTION}

There are over 1500000 major surgical procedures carried out in the UK each year, with an annual cost of about $£ 5.6$ billion. ${ }^{1}$ An increasing proportion of surgical patients are high risk, as they are elderly, frail, obese and have multiple comorbidities. Modifiable factors increase the risk of death and complications after surgery (which affect up to $75 \%$ of patients ${ }^{2}$ and reduce quality of life (QoL) $).{ }^{3-5}$ The implementation of enhanced recovery after surgery (ERAS), also known as 'fast-track surgery', has led to considerable improvements in patient care, ${ }^{6}$ although these programmes have largely focused on optimising the surgical and recovery pathways in hospital with little focus on preoperative patient optimisation.

Prehabilitation is a broad term applied to interventions administered prior to surgery to improve health and fitness with the aim of reducing surgery-related morbidity and facilitating recovery. Prehabilitation programmes include physical activity, ${ }^{7-13}$ nutrition support, ${ }^{14}$ smoking cessation, ${ }^{15}$ alcohol cessation, ${ }^{16}$ respiratory interventions (eg, incentive spirometry (IS) and inspiratory muscle 
training (IMT)), ${ }^{17}$ education ${ }^{18}$ and combined interventions. ${ }^{19}$ The inclusion of prehabilitation to standard ERAS programmes could allow patients to optimise their eligibility for surgery and further improve their outcomes. ${ }^{20}$

There are a large number of systematic reviews of prehabilitation, but most of these have focused on a single intervention for a specific surgical group (eg, exercise in cancer surgery ${ }^{21}$; IMT in cardiac surgery ${ }^{22}$; immunonutrition in head and neck surgery ${ }^{23}$; etc). This is despite the fact that risk factors for surgical complications are similar across all types of major surgery and, generally, clinicians in preoperative assessment clinics (where prehabilitation is likely to be implemented) assess and treat all patients, regardless of type of surgery patients undergo. The objectives of this systematic review were to (a) identify all interventions that have been administered prior to any major elective surgery, (b) evaluate the potential benefits and harms of these interventions, and (c) compare the effectiveness of the different interventions on postoperative outcomes.

\section{METHODS}

The protocol for this review was published previously. ${ }^{24}$ We included all published and unpublished randomised controlled trials (RCTs) with the following characteristics: (1) Participants: adult patients ( $\geq 18$ years) undergoing major elective surgery (under general anaesthesia resulting in a minimum hospital stay of at least 2 days), excluding day case surgery; (2) Interventions: administered before elective surgery with the aim of improving shortterm (up to 3 months) postoperative outcomes. Interventions administered for less than 24 hours before surgery and/or continued postoperatively, studies focusing on ERAS, studies of enteral (via nasogastric tube) or parenteral nutrition, and intravenous drug administration were excluded. Studies in which the intervention was designed to improve a functional outcome specific to one type of surgery (eg, knee exercises to improve movement of the knee after arthroplasty) were excluded, as these were not deemed to represent a generic improvement of functional capacity. The comparator was no intervention or usual care; studies comparing different prehabilitation interventions or where the comparator deviated substantially from usual care were excluded. (3) Outcomes: primary outcomes included mortality (30 days), hospital length of stay $(\mathrm{LoS})$ and postoperative complications (infective and non-infective). Secondary outcomes included LoS in intensive care unit or high dependency unit, perioperative morbidity, hospital readmission, postoperative pain, health-related quality of life (QoL), outcomes specific to the intervention, intervention-specific adverse events and resource use.

\section{Identification of studies}

The following electronic databases were searched up to June 2020, with no language restrictions: Medline and PreMedline (OvidSP) (1950 to date), Embase
Classic+Embase (OvidSP) (1974 to date), CENTRAL, DARE, HTA and NHS EED (The Cochrane Library, latest Issue), CINAHL (1981 to date), PsychINFO (1806 to date), ISI Web of Science: Science Citation Index Expanded (1900 to date), ISI Web of Science: Conference Proceedings Citation Index-Science (1990 to date), Current Controlled Trials (www.controlled-trials.com with links to other databases of ongoing trials) and the WHO International Clinical Trials Registry Platform ( www.who.int/ictrp/en/).

Reference lists of included studies and reviews were hand searched. OpenGrey, Google (to page 10), nonindexed journals, theses and dissertations, and published protocols were also searched until June 2020. Experts in the field and trial authors were contacted for further information or unpublished data. The search strategy for Medline is shown in the online supplemental digital content; this was adapted as appropriate for searching other databases.

\section{Study selection and data extraction}

One review author independently screened all titles and abstracts for eligibility (RP). A second review author screened a randomly selected sample (10\%) (MP). Two review authors (RP and MP) independently assessed all full-text papers for eligibility. Any disagreements were resolved by discussion and consensus with a third review author (CA). Reasons for excluding studies were recorded (see online supplemental digital content). Relevant data were extracted by multiple independent reviewers (each study had two independent reviewers) and all data extractions were checked and moderated by RP, MP and CA. Where multiple papers reported the same study but different outcomes, all sources were used for data extraction.

\section{Risk of bias}

Risk of bias (RoB) was independently assessed by two review authors using The Cochrane Collaboration RoB tool. ${ }^{25}$ Outcomes were grouped into outcome domains and pragmatic a priori decisions were made when assessing RoB for each of these domains. For example, lack of blinding of outcome assessors was not deemed to be able to influence objective outcomes such as mortality, so this outcome was judged to be at low risk of detection bias irrespective of blinding (see online supplemental material for RoB tool adapted for this review). The strength of the overall body of evidence for each outcome was assessed using the Grading of Recommendations, Assessment, Development and Evaluation (GRADE) methodology. ${ }^{26}$

\section{Data synthesis}

Results for trials that used variations of similar interventions (eg, different types of physical activity, psychological or educational programmes) were grouped. Due to heterogeneity in participants, interventions and intervention delivery, a random-effects meta-analysis was used for 
the primary analysis when pooling data across trials. Metaanalyses were only conducted if data from three or more trials were available. Findings from studies that were not meta-analysed were summarised narratively. Fixed-effects meta-analysis was used as a secondary analysis. Reasons for missing data were recorded (eg, drop-outs, losses to follow-up and withdrawals).

Where primary outcome data were not provided in the form of a mean and SD, we derived these from the reported test statistics (eg, SD from SEs or 95\% CIs) or estimated them (eg, mean and SD from median and range). We used the following methods to estimate or impute missing data: (1) where LoS aggregate data were presented as median and range, we estimated mean and SD using the formulae described by McGrath $e t a t^{27}$; (2) we imputed missing SDs for LoS using the mean of the SDs reported by other studies within that treatment arm; (3) where LoS data were presented as Kaplan-Meier graphs, we extracted the following LoS data for each trial arm where available: median $(50 \%)$, IQR $(25 \%-75 \%)$ and range (minimum and maximum). Mean LoS and its associated SD were subsequently derived as described above. We did not use results for LoS presented as HRs without further descriptive LoS measures to estimate median LoS, due to potentially high uncertainty in estimation ${ }^{28}$; (4) where complications were reported as per cent incidence, we converted this into the number of participants who experienced complications.

Pooled risk ratios (RRs) and 95\% CIs were calculated for dichotomous outcomes using the Mantel-Haenszel method for both random-effects and fixed-effects metaanalyses. Pooled mean differences and 95\% CIs or standardised mean differences and 95\% CI were calculated for continuous outcomes (LoS) using the inverse-variance method (for both random-effects and fixed-effects metaanalyses), when results were reported on the same scale (or could be converted to the same scale) or if results were reported on different scales, respectively. The unit of analysis in all included studies was the individual participant. No studies used cluster randomisation. Funnel plots were used to assess publication bias when 10 or more studies had been included in a meta-analysis. We formally tested for funnel plot asymmetry using the Egger's regression test. All plots are shown in the online supplemental material.

\section{Assessment of heterogeneity}

Clinical heterogeneity across studies was assessed by examining variability in participants, baseline data, interventions and outcomes. Statistical heterogeneity was quantified using the $\mathrm{I}^{2}$ statistic. We applied the following thresholds for the interpretation of the $\mathrm{I}^{2}$ statistic ${ }^{29}$ : 0\%-40\% might not be important; $30 \%-60 \%$ may represent moderate heterogeneity; $50 \%-90 \%$ may represent substantial heterogeneity; $75 \%-100 \%$ represents considerable heterogeneity.

\section{Sensitivity analyses}

We prespecified three sensitivity analyses: (1) including only trials classified as 'low risk' for random sequence generation and allocation concealment; (2) excluding studies with imputed results; (3) fixed-effects meta-analyses.

\section{Subgroup analyses}

We prespecified the following subgroup analyses: (1) type of surgery (eg, orthopaedic, cardiac, abdominal); (2) cancer versus non-cancer surgery; (3) type of intervention (eg, brief, 5 days or less vs longer term, more than 5 days); (4) intervention conducted pre-ERAS or post-ERAS implementation; (5) high-risk versus low-risk surgical patients. All analyses were performed on RevMan (V.5.3).

\section{Patient and public involvement}

No patients were involved in the design, conduct, and analysis of the study and interpretation of findings, although prehabilitation was identified as one of the priority themes for research by the James Lind Alliance Heart Surgery Priority Setting Partnership and the James Lind Alliance Anaesthesia and Perioperative Care Priority Setting Partnership, which included patient stakeholder groups.

\section{RESULTS}

One hundred ninety-four articles pertaining to 178 studies were eligible for inclusion (figure 1). Of these, 29 were unpublished studies (and 5 of these were included in meta-analyses). A summary of the interventions and main results is shown in table 1 . The characteristics of the included studies by type of intervention, aggregate data from the included studies by type of intervention, RoB assessment, summary of findings, excluded studies, studies that fit the inclusion criteria but had no usable data, and relevant protocols of ongoing or unpublished studies are shown in the online supplemental digital content. We identified eight types of interventions administered in the preoperative period: nutritional (51 studies),${ }^{30-80}$ respiratory (30 interventions of 29 studies) ${ }^{81-109}$ exercise (27 studies) ${ }^{110-136}$ multimodal (25 studies) ${ }^{100} 108$ 137-159 educational (17 studies), ${ }^{160-176}$ psychological (16 studies), ${ }^{177-192}$ smoking and alcohol cessation ( 7 studies) ${ }^{193-199}$ and pharmacological (5 studies) ${ }^{200-204}$ (table 1).

Nutritional interventions were further subdivided into standard oral nutritional supplements (ONS, 7 studies) ${ }^{30-35} 69$ oral immunonutrition supplements (19 studies) ${ }^{36-47} \quad 70-75 \quad 80$ weight loss interventions (11 studies), ${ }^{48-51} 53-5776205$ oral prebiotics and probiotics (6 studies), ${ }^{58-61} 7778$ dietary optimisation of comorbidities (3 studies) ${ }^{62-64}$ and other (5 studies, generally including administration of nutritional supplements such as fish oils, antioxidants, etc). ${ }^{65-68} 79$ Respiratory interventions were further subdivided into IMT (18 studies), ${ }^{81-95} 106108109$ IS (5 studies) $)^{90} 96-99$ and combined respiratory interventions (7 studies, involving combinations of IMT, IS, other respiratory exercises and physical exercises).${ }^{100-105} 107$ 


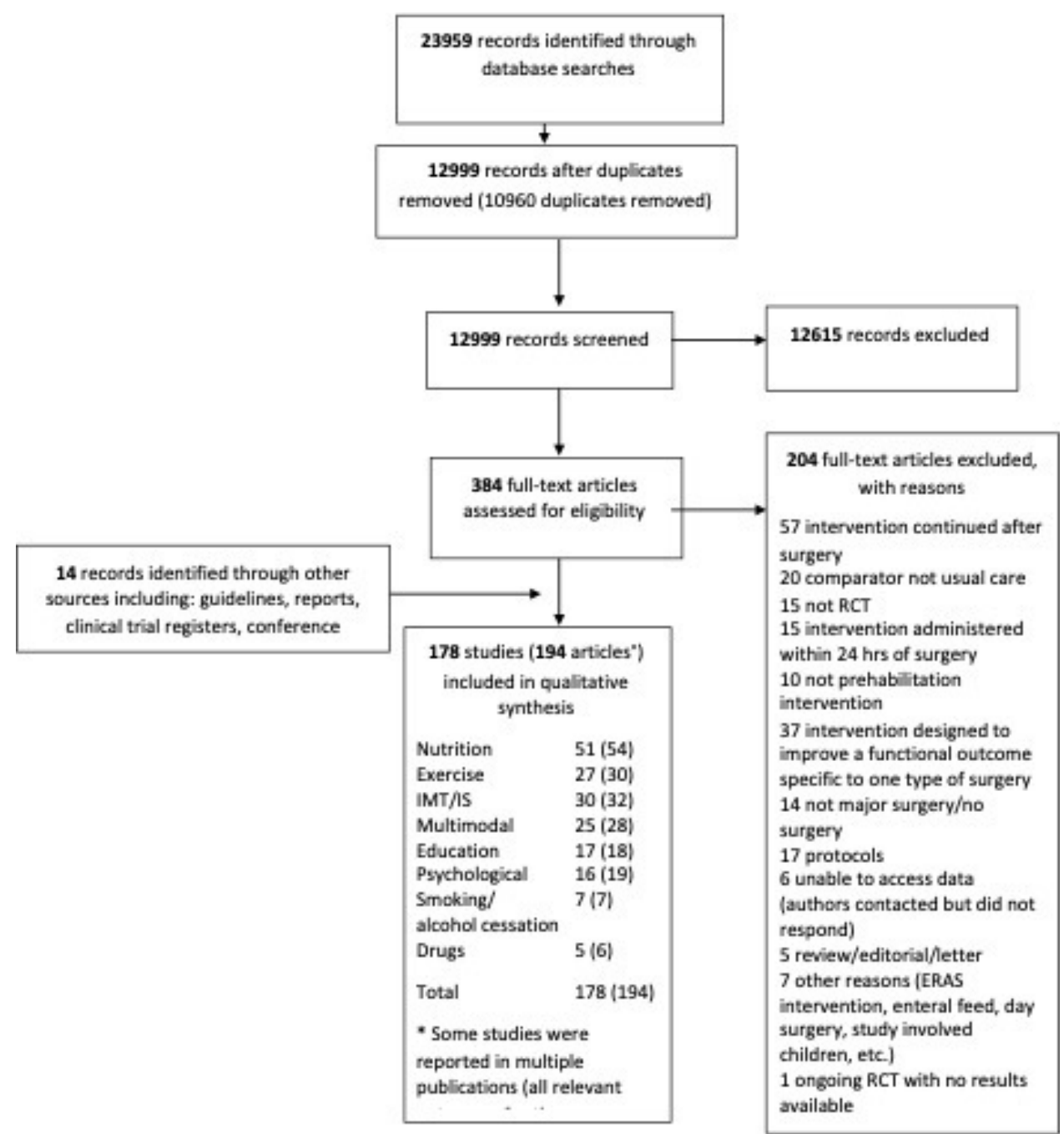

Figure 1 PRISMA ${ }^{217}$ flow diagram. ERAS, enhanced recovery after surgery; IMT, inspiratory muscle training; IS, incentive spirometry; PRISMA, Preferred Reporting Items for Systematic Reviews and Meta-Analyses; RCT, randomised controlled trial.

\section{Perioperative mortality}

All-cause perioperative mortality (approximately 30 days) reported in a format usable for pooling was available from 11 of 19 immunonutrition studies ${ }^{36-40} 45-47727480$ (910 participants); 4 of 6 ONS studies ${ }^{30-33}$ (342 participants); 3 of 6 pre/probiotics studies ${ }^{58-60}$ (214 participants); 5 of 26 exercise studies ${ }^{112} 120121126128$ (406 participants); 6 of 18 IMT studies 84869193108109 (407 participants); 3 of 16 education studies ${ }^{160} 166174$ (938 participants) and 10 of 25 multimodal studies ${ }^{108} 138140141148150151156157206$ (771 participants). Mortality rates ranged from $1 \%$ to $5 \%$. There was no effect of any prehabilitation intervention on all-cause mortality (figure 2). There was no evidence of statistical heterogeneity between studies for any intervention $\left(\mathrm{I}^{2}=0\right)$ other than ONS and pre/probiotics $\left(\mathrm{I}^{2}=33 \%\right.$ and $32 \%$, respectively). The GRADE quality of evidence ranged from low to very low.

\section{Hospital LoS}

IMT (10 of 18 studies, ${ }^{81} 85878891-931061081091003$ participants), immunonutrition (13 of 19 RCTs, ${ }^{37-41} 4345-4770727380$ 1010 participants) and multimodal interventions (18 of
25 RCTs, ${ }^{100} 108$ 137-143 145148149 151-153 1561572061529 participants) reduced LoS (figure 3 ), by -1.81 days (95\% CI -2.31 to $-1.31,-2.11$ days $(95 \%$ CI -3.07 to -1.15 ) and -1.67 days $(95 \%$ CI -2.31 to -1.03$)$, respectively. Statistical heterogeneity was moderate for immunonutrition $(31 \%)$ and high for multimodal $\left(\mathrm{I}^{2}=80 \%\right)$. The GRADE quality of evidence was moderate for IMT, low for multimodal and very low for immunonutrition.

\section{Total complications (infective and non-infective)}

Results were pooled for immunonutrition (7 of 19

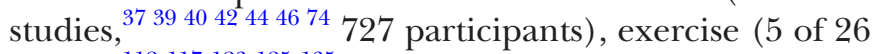
studies, ${ }^{112} 117123125135287$ participants) and multimodal (5 of 25 studies, ${ }^{138} 142150155206313$ participants) (figure 4). Multimodal interventions reduced risk of total complications by $16 \%$ (risk ratio (RR) $0.84,95 \%$ CI 0.72 to 0.97 , $\mathrm{I}^{2}=0 \%$ ). The GRADE quality of evidence was very low or low for all three interventions.

\section{Total infective complications}

Total infective complications were reported in 6 of 19 immunonutrition studies ${ }^{3638-404372}$ (609 participants), 
Table 1 Summary of prehabilitation interventions, main results and GRADE quality of evidence rating

\begin{tabular}{lll}
\hline Pre-admission intervention & $\begin{array}{l}\text { N studies identified and } \\
\text { study characteristics }\end{array}$ & Surgical populations included \\
\hline Nutritional interventions & & \\
Oral nutritional & 7 studies published between & 2 hepatectomy \\
supplements) $^{30-3569}$ & 2000 and 2018, including & 4 colorectal/Gl \\
& 23-125 ppts. & 1 oesophagogastric junction \\
& 4 UK & \\
& 2 Japancer \\
& 1 China & \\
& &
\end{tabular}

\section{Interventions}

Fortisip/Fortijuice (2 studies, 1 of

\begin{tabular}{|c|c|c|}
\hline Immunonutrition ${ }^{36-47} 70-7580$ & $\begin{array}{l}19 \text { studies published between } \\
2002 \text { and } 2020 \text {, including } \\
14-244 \text { ppts. } \\
5 \text { Western Europe } \\
4 \text { Japan } \\
2 \text { Turkey } \\
1 \text { Australia } \\
1 \text { USA } \\
1 \text { India } \\
1 \text { Lithuania } \\
1 \text { Mexico } \\
1 \text { New Zealand } \\
2 \text { not stated }\end{array}$ & $\begin{array}{l}2 \text { colorectal cancer } \\
1 \text { upper or lower GI } \\
3 \text { Gl cancer } \\
1 \text { total gastrectomy (cancer) } \\
4 \text { pancreatic cancer } \\
2 \text { any abdominal surgery } \\
1 \text { lung cancer } \\
2 \text { cardiac surgery } \\
2 \text { hepatectomy/liver cancer } \\
1 \text { enterocutaneous fistula }\end{array}$ \\
\hline
\end{tabular}
which also gave dietary advice),

Nutrison fibre (1 study), unnamed liquid formulas/oral feeds

3 studies, 1 of which also gave dietary advice), or Livact (1 study) Where stated volumes ranged from $250 \mathrm{~mL} /$ day to $1400 \mathrm{~mL} /$ day, and duration ranged from 5 days to 1 month. Oral consumption, usually self-administered at home. 3 studies reported intervention delivered by dietitian/nutritionist. Number of contacts generally not reported.

Comparator: standard diet/ no supplementation (5 studies), dietary advice (2 studies).

Most (15 studies) used combined arginine, omega-3 fatty acids, and RNA; 1 study used alanyl glutamine, 1 used L-glutamine, 1 used arginine +glutamine, and 1 used L-arginine +PUFA), and, where reported, generally ranged from $711 \mathrm{~mL} /$ day to $1 \mathrm{~L} /$ day, for 3-10 days. Where reported, usually oral consumption at home, hospital, or home and hospital. Comparator: no supplement/usual care/standard diet (17 studies), maltodextrin (1 study), NR (1 study).

Main results

Mortality RR $1.18(95 \% \mathrm{Cl}$ 0.23 to 6.11$), p=0.85$ GRADE rating: very low LoS MD $-0.54(95 \% \mathrm{Cl}$ -1.38 to 0.29$), p=0.20$ GRADE rating: very low Could not pool results for the other outcomes

Mortality RR 0.55 (95\% C 0.21 to 1.42$), p=0.22$ GRADE rating: low LoS MD $-2.11(95 \% \mathrm{Cl}$ -3.07 to -1.15$), p<0.0001$ GRADE rating: very low Total complications (infective and noninfective) RR $0.74(95 \% \mathrm{Cl}$

0.54 to 1.02$), p=0.07$ GRADE rating: very low Total infective complications RR 0.64 (95\% Cl 0.40 to 1.01) $\mathrm{p}=0.05$

GRADE rating: very low Wound infection RR 0.71 (95\% Cl 0.51 to 0.99 ) $\mathrm{p}=0.05$ GRADE rating: very low Pneumonia RR 0.52 (95\% $\mathrm{Cl} 0.18$ to 1.44$), p=0.21$ GRADE rating: very low

LoS MD 0.22 (95\% C -0.46 to 0.91 ), $\mathrm{p}=0.53$ GRADE rating: very low iquid diet or ppt-led calorie restriction (with/without diet sheets). Where reported, length of Could not pool results for time on diet ranged from 3 days to 8 weeks (in 4 studies this was 14 days).

All home-based interventions; some (5 studies) delivered by dietitian/nutritionist and some (6 studies) reported number of contacts (range 1-3 times or described as 'regular phone calls'). Comparator was mostly usual diet or standard care (note that standard diet in one study was $1000 \mathrm{kcal} /$ day low-carbohydrate, high-protein diet). 
Table 1 Continued

\begin{tabular}{|c|c|c|c|c|}
\hline Pre-admission intervention & $\begin{array}{l}\text { N studies identified and } \\
\text { study characteristics }\end{array}$ & Surgical populations included & Interventions & Main results \\
\hline Pre/probiotics ${ }^{58-617778}$ & $\begin{array}{l}6 \text { studies published between } \\
2004 \text { and } 2019, \text { including } \\
55-137 \text { ppts. } \\
3 \text { Europe } \\
2 \text { Brazil } \\
1 \text { China }\end{array}$ & $\begin{array}{l}5 \text { colorectal resection/ } \\
\text { surgery for colorectal cancer } \\
\text { or elective laparotomy } \\
\text { (predominantly colectomy) } \\
1 \text { liver transplant }\end{array}$ & $\begin{array}{l}\text { Five different formulations: } \\
\text { Probiotic capsule +prebiotic } \\
\text { (oligofructose) } \\
\text { Lyophilised yeast } \\
\text { capsule +Saccharomyces boulardi } \\
\text { ProBacti } 4 \text { Enteric (Lactococcus } \\
\text { lactis, Lactobacillus casei, L. } \\
\text { acidopholous, and Bifidobacterium } \\
\text { bifidum) Simbioflora } \\
\text { (fructooligosaccharide, L. } \\
\text { acidophilus NCFM, L. rhamnosus } \\
\text { HN001, L. casei LPC-37, and B. } \\
\text { lactis HN019) } \\
\text { Synbiotic } 2000 \text { FORTE (lactic acid } \\
\text { bacteria, Pediacoccus pentosaceus } \\
\text { and Leuconostoc mesenteroides) } \\
\text { Oral bifid triple viable capsules } \\
\text { (B. longum, L. acidophilus and } \\
\text { Enterococcus faecalis) } \\
\text { Usually home-based oral } \\
\text { intervention for } 3 \text { or } 7 \text { days (not } \\
\text { stated in two studies). } 2 \text { studies } \\
\text { reported daily phone calls (number } \\
\text { of contacts not stated for others). } \\
\text { Comparator was placebo } \\
\text { capsules/powder ( } 4 \text { studies) or } \\
\text { usual care ( } 2 \text { studies). }\end{array}$ & $\begin{array}{l}\text { Mortality } \\
\text { RR=0.76 (95\% Cl } 0.17 \text { to } \\
3.42), p=0.72 \\
\text { GRADE rating: low } \\
\text { Total PO infective } \\
\text { complications } \\
\text { RR } 0.48 \text { ( } 95 \% \mathrm{Cl} 0.14 \text { to } \\
1.62), p=0.23 \\
\text { GRADE rating: low } \\
\text { Could not pool results for } \\
\text { the other outcomes }\end{array}$ \\
\hline Nutritional optimisation ${ }^{62-64}$ & $\begin{array}{l}3 \text { studies published between } \\
1987 \text { and } 2014 \text { including } \\
35-41 \text { ppts. } \\
1 \text { UK } \\
1 \text { USA } \\
1 \text { not stated }\end{array}$ & $\begin{array}{l}1 \text { Roux-en-Y gastric bypass } \\
1 \text { upper GI } \\
1 \text { elective } C A B G\end{array}$ & $\begin{array}{l}\text { Optimisation of glucose or general } \\
\text { nutrition, or low glycaemic index } \\
\text { diet. Duration ranged from } 10 \text { days } \\
\text { to } 3 \text { months. Where reported, } \\
\text { generally oral/written advice (one } \\
\text { or two contacts), self-delivered at } \\
\text { home. } \\
\text { Comparator was nutrition } \\
\text { counselling, or high glycaemic } \\
\text { index diet (not stated in one study). }\end{array}$ & $\begin{array}{l}\text { No meta-analyses } \\
\text { conducted on any outcome } \\
\text { due to limited data }\end{array}$ \\
\hline $\begin{array}{l}\text { Other nutritional } \\
\text { interventions }\end{array}$ & $\begin{array}{l}5 \text { studies published between } \\
2007 \text { and } 2019, \text { including } \\
30-105 \text { ppts. } \\
2 \text { Europe } \\
1 \text { India } \\
1 \text { Australia } \\
1 \text { Iran }\end{array}$ & $\begin{array}{l}3 \text { CABG/cardiac } \\
1 \text { lung cancer } \\
1 \text { lumbar spine }\end{array}$ & $\begin{array}{l}\text { Five different interventions: } \\
\text { Combined supplement (glutamine, } \\
\text { L-carnitine, vitamins C, E, and } \\
\text { selenium) } \\
\text { Vitamin D } \\
\text { Coenzyme Q10 } \\
\text { a-ketoglutaric acid and } \\
\text { 5-hydroxymethylfurfural } \\
\text { Fish oil } \\
\text { Oral consumption, usually at home, } \\
\text { for } 7 \text { days-5 weeks. Comparator } \\
\text { was placebo ( } 2 \text { studies) or no } \\
\text { supplement/usual nutrition/usual } \\
\text { care ( } 3 \text { studies) } \\
\text { None reported the number of } \\
\text { contacts. }\end{array}$ & $\begin{array}{l}\text { No meta-analyses } \\
\text { conducted on any outcome } \\
\text { due to limited data and } \\
\text { because of intervention } \\
\text { heterogeneity }\end{array}$ \\
\hline Exercise $^{110-126128129131-136219}$ & $\begin{array}{l}27 \text { studies published between } \\
1996 \text { and } 2020, \text { including } \\
14-164 \text { ppts. } \\
1 \text { Russia } \\
7 \text { UK } \\
1 \text { Ireland } \\
6 \text { Western Europe } \\
3 \text { Canada } \\
2 \text { Australia } \\
2 \text { USA } \\
1 \text { Japan } \\
1 \text { Brazil } \\
3 \text { not stated }\end{array}$ & $\begin{array}{l}1 \text { cardiac } \\
1 \text { radical cystectomy } \\
2 \text { AAA } \\
1 \text { bariatric } \\
6 \text { TKR/THR//THA/TKA } \\
2 \text { liver resection } \\
3 \text { lung cancer } \\
1 \text { radical prostatectomy } \\
1 \text { abdominal surgery } \\
2 \text { lumbar spine surgery } \\
1 \text { thoracic surgery } \\
1 \text { urological surgery } \\
1 \text { rectal cancer } \\
2 \text { colorectal surgery } \\
1 \text { cardiac or thoracic surgery } \\
1 \text { knee osteoarthritis }\end{array}$ & $\begin{array}{l}\text { Different protocols involving } \\
\text { different amounts of cardio } \\
\text { and strengthening exercises } \\
\text { in both group and individual } \\
\text { format. Individual programmes } \\
\text { often tailored, for example, } \\
\text { physiotherapy. Many studies } \\
\text { reported supervision. Specific } \\
\text { intensity sometimes mentioned, } \\
\text { eg, moderate, HIIT. Length of } \\
\text { intervention: } 26 \text { studies: } 1-8 \\
\text { weeks; } 1 \text { study: } 15-17 \text { weeks. } \\
\text { Comparator: usual care ( } 22 \\
\text { studies), usual care and diet } \\
\text { therapy (1 study), usual care and } \\
\text { additional exercise regimen (1 } \\
\text { study), NR ( } 2 \text { studies). }\end{array}$ & $\begin{array}{l}\text { Mortality RR } 0.74(95 \% \mathrm{Cl} \\
0.23 \text { to } 2.35), \mathrm{p}=0.61 \\
\text { GRADE rating: low } \\
\text { LoS } \mathrm{MD}-0.38 \text { days }(95 \% \mathrm{Cl} \\
\text {-0.82 to } 0.06), \mathrm{p}=0.09 \\
\text { GRADE rating: very low } \\
\text { Total PO complications } \\
\text { RR } 0.83 \text { ( } 95 \% \mathrm{Cl} 0.61 \text { to } \\
1.12), \mathrm{p}=0.22 \\
\text { GRADE rating: low } \\
\text { Pneumonia } \\
\text { RR } 0.72 \text { (95\% Cl } 0.35 \text { to } \\
\text { 1.44), } \mathrm{p}=0.35 \\
\text { GRADE rating: very low } \\
\text { PPCs RR } 0.54 \text { ( } 95 \% \mathrm{Cl} 0.39 \\
\text { to } 0.75), \mathrm{p}=0.0003 \\
\text { GRADE rating: low }\end{array}$ \\
\hline
\end{tabular}


Table 1 Continued

\begin{tabular}{|c|c|c|c|c|}
\hline Pre-admission intervention & $\begin{array}{l}\mathrm{N} \text { studies identified and } \\
\text { study characteristics }\end{array}$ & Surgical populations included & Interventions & Main results \\
\hline $\begin{array}{l}\text { Inspiratory muscle training } \\
(\text { IMT) })^{81-8890-95106108109220}\end{array}$ & $\begin{array}{l}18 \\
\text { studies published between } \\
1996 \text { and } 2020 \text {, including } \\
16-279 \text { ppts. } \\
7 \text { Western Europe } \\
1 \text { UK } \\
1 \text { Eastern Europe } \\
4 \text { Brazil } \\
3 \text { China } \\
1 \text { Israel } \\
1 \text { not stated }\end{array}$ & $\begin{array}{l}7 \text { cardiac } \\
3 \text { thoracic (lung cancer) } \\
1 \text { abdominal aortic aneurysm } \\
1 \text { bariatric } \\
1 \text { laparoscopic bariatric } \\
1 \text { colorectal cancer } \\
1 \text { THR } \\
1 \text { abdominal or urological } \\
2 \text { oesophagectomy }\end{array}$ & $\begin{array}{l}\text { Threshold IMT starting at } 30 \%- \\
40 \% \text { MIP } 4 \times / \text { day to } 2 \times / \text { week (but } \\
\text { most at least once daily) for } 4 \\
\text { days } 4 \text { weeks before surgery (most } \\
\text { for } 2 \text { weeks). Most included weekly } \\
\text { contact with ppts. } \\
\text { Comparator usual care; only } 1 \\
\text { study included a sham IMT training } \\
\text { group as a comparator. }\end{array}$ & $\begin{array}{l}\text { Mortality RR } 1.49 \text { ( } 95 \% \mathrm{Cl} \\
0.60 \text { to } 3.69), \mathrm{p}=0.39 \\
\text { GRADE rating: low } \\
\text { LoS MD }-1.81 \text { days }(95 \% \mathrm{Cl} \\
-2.31 \text { to }-1.32), \mathrm{p}<0.00001 \\
\text { GRADE rating: moderate } \\
\text { PPCs } \mathrm{RR} 0.55 \text { ( } 95 \% \mathrm{Cl} 0.38 \\
\text { to } 0.80), \mathrm{p}=0.002 \\
\text { GRADE rating: low } \\
\text { Pneumonia RR } 0.69(95 \% \\
\mathrm{Cl} 0.49 \text { to } 1.05), \mathrm{p}=0.08 \\
\text { GRADE rating: very low }\end{array}$ \\
\hline Incentive spirometry (IS) $)^{9096-99}$ & $\begin{array}{l}5 \text { studies published between } \\
1983 \text { and } 2014, \text { including } \\
41-172 \text { ppts. } \\
2 \text { USA } \\
1 \text { UK } \\
1 \text { Brazil } \\
1 \text { not stated }\end{array}$ & $\begin{array}{l}1 \text { cardiac } \\
1 \text { THR } \\
1 \text { abdominal (type not stated) } \\
1 \text { laparoscopic (bariatric) }\end{array}$ & $\begin{array}{l}\text { IS (different protocols, generally } \\
4-10 \text { repetitions/day) for } 1 \text { week } \\
\text { before surgery. } \\
\text { Comparator usual care; } 1 \text { study } \\
\text { included sham IS. }\end{array}$ & $\begin{array}{l}\text { LoS MD }-2.39(95 \% \mathrm{Cl} \\
-5.50 \text { to } 0.72), \mathrm{p}=0.13 \\
\text { GRADE rating: very low } \\
\text { PPCs RR } 0.68 \text { ( } 95 \% \mathrm{Cl} 0.25 \\
\text { to } 1.81), \mathrm{p}=0.44 \\
\text { GRADE rating: very low } \\
\text { No meta-analyses } \\
\text { conducted on any other } \\
\text { outcome due to limited data }\end{array}$ \\
\hline $\begin{array}{l}\text { Combined respiratory } \\
\text { interventions }\end{array}$ & $\begin{array}{l}\text { 7studies } \\
\text { published between } 1998 \text { and } \\
2018 \text {, including 9-60 ppts. } \\
3 \text { Spain } \\
1 \text { USA } \\
\text { 1 Turkey } \\
\text { 2not stated }\end{array}$ & $\begin{array}{l}1 \text { thoracic (lung cancer) } \\
2 \text { cardiac (ppts with COPD) } \\
1 \text { oesophagectomy } \\
3 \text { laparoscopic bariatric }\end{array}$ & $\begin{array}{l}\text { Respiratory rehabilitation } \\
\text { (multimodal intervention designed } \\
\text { for people with impaired lung } \\
\text { function; includes physical } \\
\text { exercises, breathing exercises, } \\
\text { education, etc) for } 1-4 \text { weeks } \\
\text { before surgery. } \\
\text { Chest physiotherapy programmes } \\
\text { (combinations of IS, IMT and lung } \\
\text { re-expansion). } \\
\text { Comparator usual care. }\end{array}$ & $\begin{array}{l}\text { No meta-analyses } \\
\text { conducted on any outcome } \\
\text { due to limited data }\end{array}$ \\
\hline Combined interventions $^{100} 108$ & $\begin{array}{l}25 \text { studies published between } \\
2000 \text { and } 2019 \text { including } \\
14-249 \text { ppts. } \\
3 \text { Western Europe } \\
8 \text { USA/Canada } \\
5 \text { China/Taiwan } \\
1 \text { Turkey } \\
1 \text { Egypt } \\
1 \text { Australia } \\
1 \text { Hungary } \\
5 \text { not stated }\end{array}$ & $\begin{array}{l}8 \text { lung resection } \\
3 \text { CABG } \\
2 \text { oesophagogastric resection } \\
1 \text { pancreaticoduodenectomy } \\
2 \text { colorectal cancer } \\
1 \text { prostatectomy } \\
1 \text { cystectomy } \\
4 \text { THR/TKR } \\
2 \text { elective abdominal surgery } \\
1 \text { cardiac/thoracic }\end{array}$ & $\begin{array}{l}\text { Interventions combined } 2-4 \\
\text { different modes. All studies } \\
\text { included physical activity, } 10 \\
\text { included breathing exercises, } \\
5 \text { education, } 7 \text { nutrition, } 8 \\
\text { psychological and } 1 \text { drug } \\
\text { optimisation. The most common } \\
\text { combinations were physical } \\
\text { activity +breathing ( } 6 \text { studies) and } \\
\text { physical activity +education (5 } \\
\text { studies). } \\
\text { Comparator usual care; } 1 \text { study } \\
\text { used a standard exercise +nutrition } \\
\text { protocol and a standard nutrition } \\
\text { protocol. }\end{array}$ & $\begin{array}{l}\text { Mortality RR } 0.67(95 \% \mathrm{Cl} \\
0.23 \text { to } 1.95), \mathrm{p}=0.46 \\
\text { GRADE rating: low } \\
\text { LoS } \mathrm{MD}-1.67 \text { days }(95 \% \mathrm{Cl} \\
-2.31 \text { to }-1.03), \mathrm{p}<0.00001 \\
\text { GRADE rating: moderate } \\
\text { Pneumonia } \mathrm{RR} 0.56 \text { ( } 95 \% \\
\mathrm{Cl} 0.28 \text { to } 1.12), \mathrm{p}=0.10 \\
\text { GRADE rating: very low } \\
\text { Total PO complications } \\
\text { RR } 0.84 \text { ( } 95 \% \mathrm{Cl} 0.72 \text { to } \\
0.97), \mathrm{p}=0.02 \\
\text { GRADE rating: very low }\end{array}$ \\
\hline Education $^{160-167}$ 169-176 221 & $\begin{array}{l}17 \text { studies published between } \\
1996 \text { and } 2020 \text { including } \\
35-441 \text { ppts. } \\
6 \text { USA/Canada } \\
4 \text { Western Europe } \\
2 \text { Finland } \\
2 \text { Australia } \\
1 \text { Serbia } \\
2 \text { Turkey }\end{array}$ & $\begin{array}{l}4 \text { cardiac } \\
1 \text { laparoscopic } \\
\text { cholecystectomy } \\
1 \text { unspecified abdominal } \\
1 \text { spinal surgery } \\
8 \text { THR/TKR } \\
1 \text { arthroscopic rotor cuff } \\
\text { repair } \\
1 \text { unspecified }\end{array}$ & $\begin{array}{l}\text { Structured education of different } \\
\text { levels of intensity delivered through } \\
\text { interviews and written information } \\
\text { ( } 7 \text { studies), classes and written } \\
\text { information ( } 4 \text { studies), interview } \\
\text { and website ( } 1 \text { study), education } \\
\text { session only ( } 1 \text { study), booklet, } \\
\text { interview and telephone call (1 } \\
\text { study), DVD and telephone call } \\
\text { ( } 1 \text { study), telephone call only ( } 1 \\
\text { study), education booklet only ( } 3 \\
\text { studies). Education consisted of } \\
\text { information on what to expect from } \\
\text { surgery, teaching exercises for } \\
\text { use postoperatively and the use } \\
\text { of aids. } \\
\text { Comparator usual preoperative } \\
\text { care and explanations (delivered } \\
\text { verbally, in written form and via } \\
\text { video). } 1 \text { study compared intensive } \\
\text { education sessions with practical } \\
\text { classes with a physiotherapist. }\end{array}$ & $\begin{array}{l}\text { Mortality RR } 0.83(95 \% \mathrm{Cl} \\
0.24 \text { to } 2.95), \mathrm{p}=0.78 \\
\text { GRADE rating: low } \\
\text { LoS MD } 0.00 \text { days }(95 \% \mathrm{Cl} \\
-0.40 \text { to } 0.40), \mathrm{p}=1.00 \\
\text { GRADE rating: very low } \\
\text { No meta-analyses } \\
\text { conducted on any other } \\
\text { outcome due to limited data } \\
\end{array}$ \\
\hline
\end{tabular}


Table 1 Continued

\begin{tabular}{|c|c|c|c|c|}
\hline Pre-admission intervention & $\begin{array}{l}\text { N studies identified and } \\
\text { study characteristics }\end{array}$ & Surgical populations included & Interventions & Main results \\
\hline Psychological ${ }^{177-192}$ & $\begin{array}{l}16 \text { studies published between } \\
1986 \text { and } 2020 \text { including } \\
24-400 \text { ppts. } \\
8 \text { Western Europe } \\
2 \text { US } \\
1 \text { China } \\
1 \text { Nigeria } \\
1 \text { Pakistan } \\
1 \text { Columbia } \\
2 \text { not stated }\end{array}$ & $\begin{array}{l}3 \text { cardiac (one with additional } \\
\text { poorly controlled risk factor) } \\
2 \text { abdominal (type not stated) } \\
1 \text { radical prostatectomy } \\
1 \text { colorectal cancer } \\
1 \text { cholecystectomy } \\
1 \text { bariatric } \\
2 \text { lumbar fusion } \\
1 \text { general surgery } \\
1 \text { pancreatic surgery } \\
1 \text { knee replacement surgery } \\
2 \text { varied or unspecified } \\
\text { elective surgery }\end{array}$ & $\begin{array}{l}\text { Different protocols involving } \\
\text { psychological therapies: } \\
\text { expectation management; } \\
\text { relaxation exercises; breathing } \\
\text { exercises; guided imagery; } \\
\text { mindfulness; stress management; } \\
\text { counselling; } 7 \text { studies involved } \\
\text { cognitive-behavioural therapy. } 4 \\
\text { stated that a psychologist delivered } \\
\text { the intervention. } \\
\text { Comparator majority were usual } \\
\text { care; } 1 \text { study used a control topic; } \\
3 \text { studies gave information about } \\
\text { surgical procedures or general } \\
\text { advice; } 1 \text { study offered a hospital } \\
\text { helpline number; } 1 \text { study an } \\
\text { information session with ppt and } \\
\text { support person. }\end{array}$ & $\begin{array}{l}\text { LoS MD }-0.82 \text { days }(95 \% \mathrm{Cl} \\
-1.83 \text { to } 0.19), \mathrm{p}=0.11 \\
\text { GRADE rating: very low } \\
\text { No meta-analyses } \\
\text { conducted on any other } \\
\text { outcome due to limited data }\end{array}$ \\
\hline
\end{tabular}

\begin{tabular}{|c|c|c|c|c|}
\hline Smoking cessation $^{193-197}$ & $\begin{array}{l}5 \text { studies published between } \\
2004 \text { and } 2014 \text {, including } \\
28-168 \text { ppts. } \\
2 \text { Western Europe } \\
1 \text { USA/Canada } \\
1 \text { Australia } \\
1 \text { China }\end{array}$ & $\begin{array}{l}1 \text { lower or upper fracture } \\
1 \text { THR } \\
1 \text { not stated } \\
2 \text { general surgery }\end{array}$ & $\begin{array}{l}\text { Majority used a combination of } \\
\text { medication (nicotine replacement/ } \\
\text { bupropion) and/or advice giving/ } \\
\text { counselling (face-to-face or } \\
\text { telephone) for 4-8 weeks before } \\
\text { surgery. } \\
\text { Comparator usual care. }\end{array}$ & $\begin{array}{l}\text { Wound infection } \\
R R=0.28(95 \% \mathrm{Cl} 0.12 \text { to } \\
0.64), p=0.002 \\
\text { GRADE rating: very low }\end{array}$ \\
\hline Alcohol cessation $^{199222}$ & $\begin{array}{l}2 \text { studies published in } 1999 \\
\text { and } 2002 \text {, including } 42 \text { and } 28 \\
\text { ppts., respectively } \\
\text { Both Western Europe }\end{array}$ & $\begin{array}{l}1 \text { colorectal } \\
1 \text { hip arthroplasty }\end{array}$ & $\begin{array}{l}\text { Withdrawal from alcohol, } \\
\text { motivational counselling and } \\
\text { treatment with disulfiram for 1-3 } \\
\text { months before surgery. } \\
\text { Comparator usual care. }\end{array}$ & $\begin{array}{l}\text { No meta-analyses } \\
\text { conducted on any outcome } \\
\text { due to limited data }\end{array}$ \\
\hline Pharmacological $^{200-204}$ & $\begin{array}{l}5 \text { studies published between } \\
2005 \text { and } 2020 \text {, including } \\
\text { between } 4 \text { and } 400 \text { ppts. } \\
3 \text { Western Europe } \\
1 \text { Iran } \\
\text { I Australia }\end{array}$ & $\begin{array}{l}2 \text { CABG } \\
1 \text { cardiac surgery } \\
1 \text { prostatectomy } \\
1 \text { major non-cardiac surgery }\end{array}$ & $\begin{array}{l}\text { Intervention: } \\
\text { Provision of drugs (serenoa } \\
\text { repens (Permixon); atorvastatin; } \\
\text { bisoprolol titration; allopurinol and } \\
\text { vitamin E supplement); optimising } \\
\text { dosage; management and control } \\
\text { of risk factors; } 1 \text { nephrologist } \\
\text { management; } 1 \text { nurse-led strategy. } \\
\text { Duration of intervention: } 3-5 \text { days - } \\
2 \text { months before surgery. } \\
\text { Comparator: } 4 \text { usual care; } 1 \\
\text { placebo }\end{array}$ & $\begin{array}{l}\text { No meta-analyses } \\
\text { conducted on any outcome } \\
\text { due to limited data }\end{array}$ \\
\hline
\end{tabular}

CABG, coronary artery bypass graft; COPD, chronic obstructive pulmonary disease; GI, gastrointestinal; GRADE, Grading of Recommendations, Assessment, Development and Evaluation; HIIT, high intensity interval training; LoS, length of stay; MD, mean difference; MIP, maximum inspiratory pressure; NR, not reported; PO, postoperative; PPCs, postoperative pulmonary complications; ppts, participants; PUFA, polyunsaturated fatty acids; RR, risk ratio; THA, total hip arthroplasty; THR, total hip replacement; TKA, total knee arthroplasty; TKR, total knee replacement.

and 3 of 6 pre/probiotic studies ${ }^{58-60}$ (214 participants). Immunonutrition and pre/probiotic interventions reduced the risk of infective complications by $36 \%$ (RR
$0.64,95 \%$ CI 0.40 to $\left.1.01, I^{2}=56 \%\right)$ and $52 \%$ (RR 0.48 , $95 \%$ CI 0.14 to $\left.1.62, I^{2}=66 \%\right)$, respectively. The GRADE

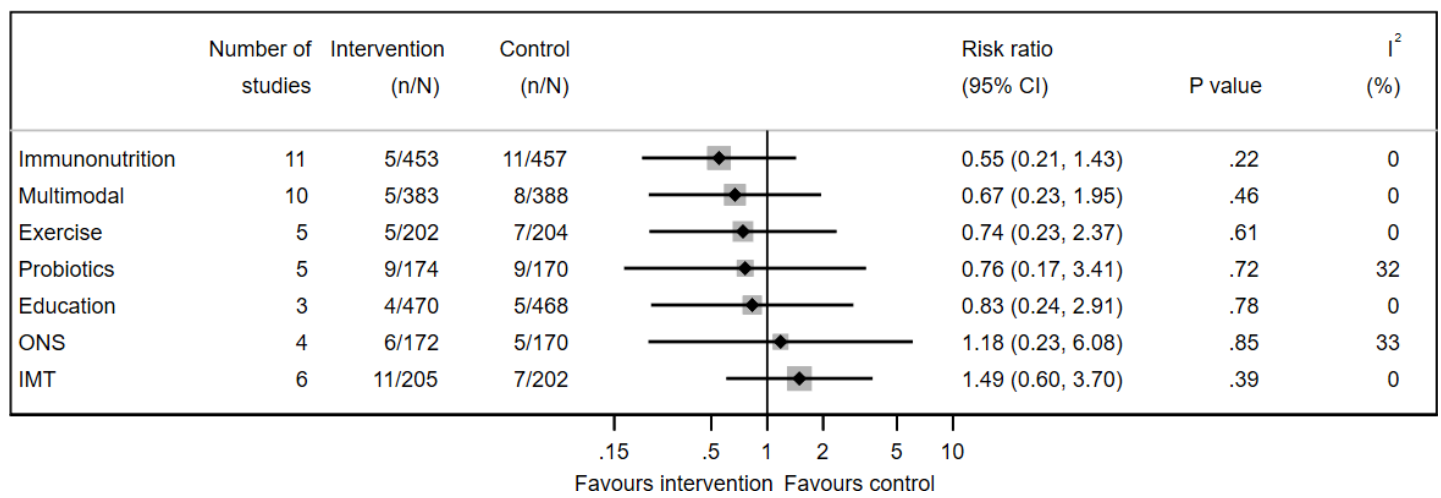

Figure 2 Forest plot of prehabilitation for reducing all-cause perioperative mortality. All interventions were tested with usual care as control. IMT, inspiratory muscle training; ONS, oral nutritional supplements. 


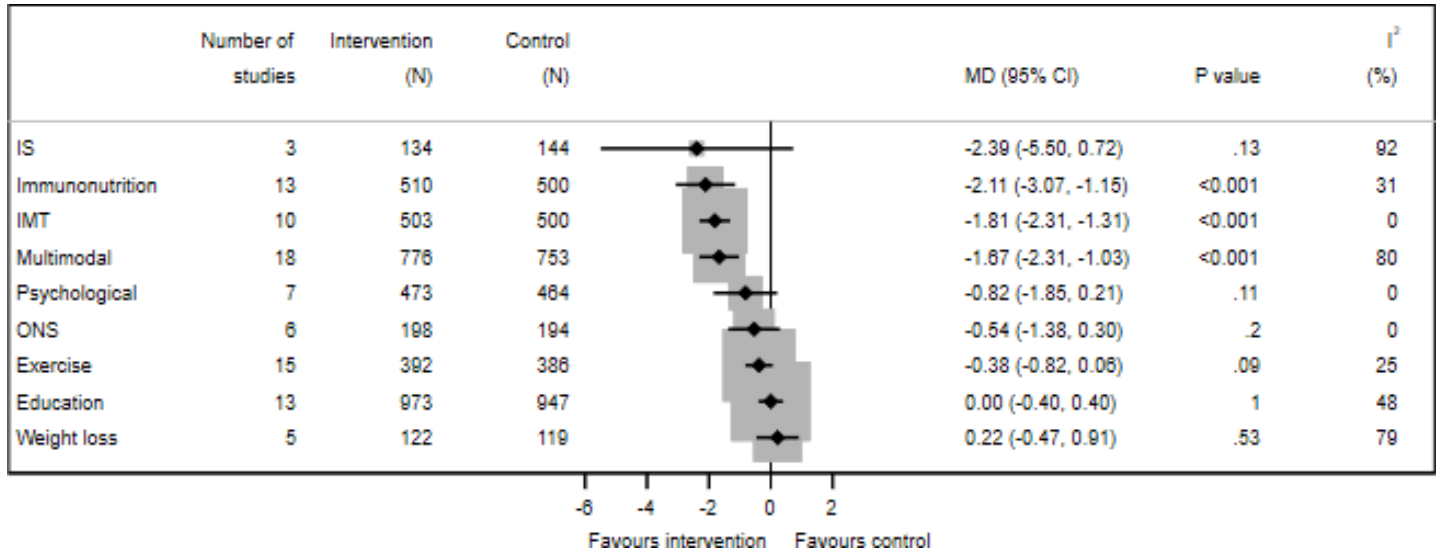

Figure 3 Forest plot of prehabilitation for reducing length of hospital stay. All interventions were tested with usual care as control. IMT, inspiratory muscle training; IS, incentive spirometry; MD, mean difference; ONS, oral nutritional supplements.

quality of evidence was very low/low, respectively (see online supplementary digital content for plots).

\section{Wound infection}

Wound infection was reported in 8 of 19 immunonutrition studies ${ }^{36} 3739-41707380$ (752 participants) and 3 of 4 smoking cessation studies ${ }^{195-197}$ (236 participants). Immunonutrition reduced wound infection by $29 \%$ (RR 0.71 , $95 \%$ CI 0.51 to $0.99, \mathrm{I}^{2}=6 \%$ ). Smoking cessation reduced wound infection by $72 \%$ (RR $0.28,95 \%$ CI 0.12 to 0.64 , $\left.\mathrm{I}^{2}=12 \%\right)$. The GRADE quality of evidence was very low for both interventions (see online supplementary digital content for plots).

\section{Postoperative pulmonary complications}

Postoperative pulmonary complications (PPCs) were reported in 5 of 18 IMT studies 81858795106 (633 participants), 4 of 5 IS studies ${ }^{96-99}$ (315 participants) and 4 of 26 exercise studies ${ }^{112} 118121126$ (325 participants). IMT and exercise interventions reduced PPCs by $45 \%$ and $46 \%$ (RR $0.55,95 \%$ CI 0.38 to $0.80, \mathrm{I}^{2}=18 \%$ and RR 0.54 , $95 \%$ CI 0.39 to $0.75, \mathrm{I}^{2}=0 \%$ ), respectively (figure 5 ). GRADE quality of evidence was low for exercise and IMT and very low for IS.

\section{Pneumonia}

Pneumonia was reported in 11 of 18 IMT

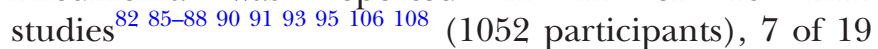
immunonutrition studies 36394142707280 (521 participants), 4 of 26 exercise studies ${ }^{111} 121126207$ (266 participants) and 7 of 20 multimodal studies ${ }^{100} 108137148149151157208$ (341 participants) (figure 6). IMT reduced the risk of pneumonia by $31 \%$ (RR $0.69,95 \%$ CI 0.46 to $1.04, \mathrm{I}^{2}=18 \%$ ). The GRADE quality of evidence for pneumonia was very low for all interventions.

\section{Funnel plots}

Funnel plots were constructed for immunonutrition, IMT, multimodal, exercise and educational interventions for LoS only, as these were the only outcome that had $>10$ studies contributing to the meta-analyses for each intervention. None of these funnel plots showed marked asymmetry (see online supplemental digital content). The Egger's regression tests confirmed that there was no marked asymmetry for any intervention $(\mathrm{p}<0.05)$, except for multimodal $(\mathrm{p}=0.01)$, suggesting that for most of the results reporting biases (including publication bias) are unlikely to be an issue. However, for some interventions (eg, immunonutrition), the low power and heterogeneity of the included studies may limit the conclusion that can be drawn from the funnel plots. ${ }^{209}$ We did not construct a funnel plot for immunonutrition and the mortality outcome because although we had 10 studies contributing data to this outcome, 4 of these included zero events.

\section{Sensitivity analyses}

None of the sensitivity analyses conducted materially altered the results of the main analyses for any intervention or outcome, except for immunonutrition and the LoS outcome. When including only RCTs that had low RoB for random sequence generation and allocation

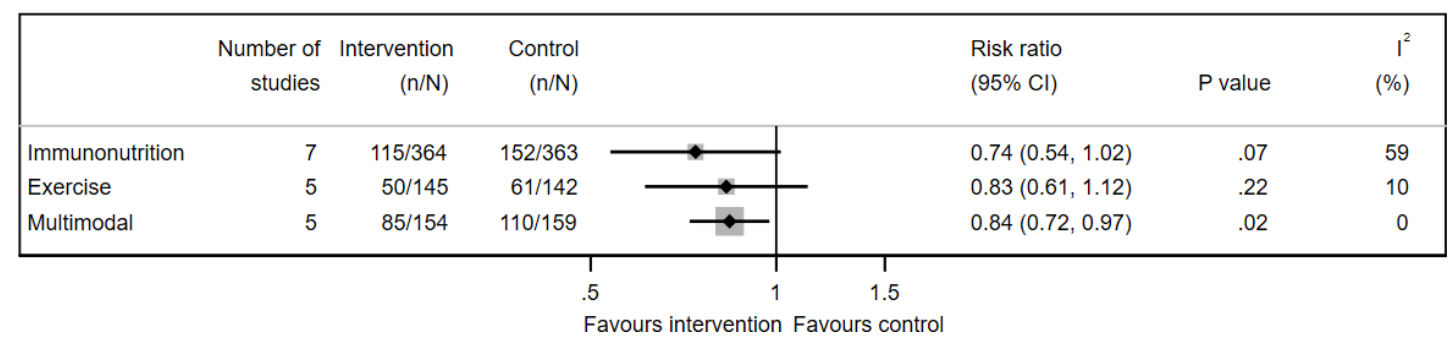

Figure 4 Forest plot of prehabilitation for reducing total postoperative complications. All interventions were tested with usual care as control. 


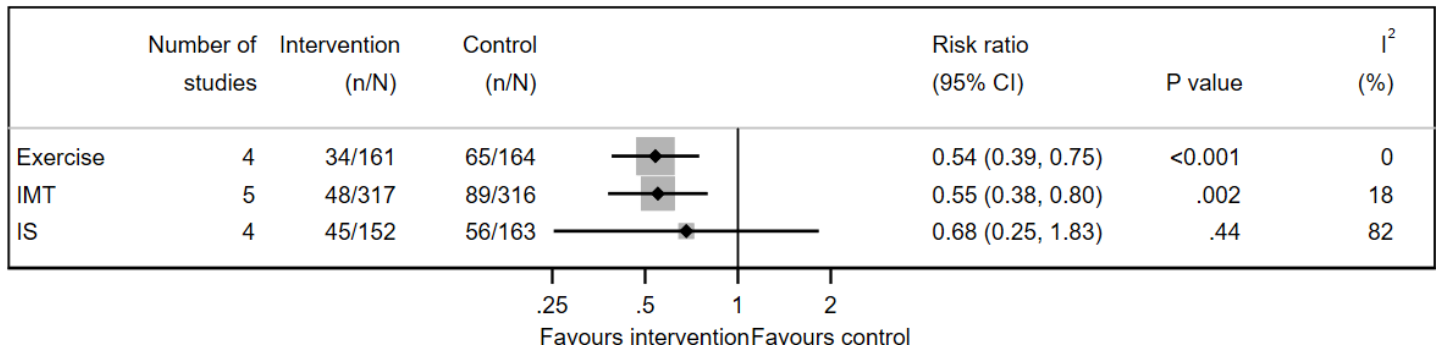

Figure 5 Forest plot of prehabilitation for reducing postoperative pulmonary complications. All interventions were tested with usual care as control. IMT, inspiratory muscle training; IS, incentive spirometry.

concealment, the pooled estimate was attenuated (immunonutrition: -1.14 days, $95 \% \mathrm{CI}-2.69$ to $0.41, \mathrm{p}=0.15)$. Estimates from all fixed-effects meta-analyses were similar to those from the random-effects meta-analyses. All sensitivity analyses are shown in the online supplemental digital content.

\section{Subgroup analyses}

Of the five prespecified subgroup analyses, we pooled data for type of surgery. Although none of the studies reported having ERAS protocols as part of usual care, we performed a subgroup analysis by grouping studies published before and after 2010, when ERAS programmes began to be implemented. We could not do subgroup analysis for the other three prespecified subgroups.

Subgroup analyses by type of surgery were conducted for cancer surgery (immunonutrition, exercise and multimodal interventions), orthopaedic surgery (multimodal interventions) and cardiac surgery (IMT). In people undergoing cancer surgery, immunonutrition ${ }^{38-41} 4380$ and multimodal interventions ${ }^{100} 138142148149151206208$ reduced LoS by about 2 days (mean difference (MD) $-1.83,95 \%$ CI -2.85 to -0.80 ) and 2.5 days (MD -2.50 days, $95 \% \mathrm{CI}$ -4.05 to -0.95$)$, respectively, while exercise interventions ${ }^{111} 120121125135$ did not reduce LoS (MD -0.16 days, $95 \% \mathrm{CI}-0.63$ to 0.31 ). In people undergoing knee/hip replacement surgery, multimodal interventions ${ }^{141} 143145$ reduced $\operatorname{LoS}$ by about 1 day (MD $-1.43,95 \% \mathrm{CI}-2.84$ to -0.02 ).

In people undergoing cardiac surgery, IMT 85878892106 reduced $\operatorname{LoS}(\mathrm{MD}-1.73$ days, $95 \%$ CI -2.39 to -1.07 ) and pneumonia ${ }^{82} 86-8895$ (RR $0.53,95 \%$ CI 0.39 to 0.73 ). None of the interventions reduced mortality in the above surgical subgroups.
Subgroup analyses including studies published before or after 2010 did not change any of our findings; effect size and direction were similar for all the interventions and outcomes that could be grouped, although some did not reach statistical significance because of small sample size. All subgroup analyses are shown in the online supplemental digital content.

\section{DISCUSSION}

The main findings from this review are that four types of prehabilitation-IMT, exercise, immunonutrition and multimodal-reduced postoperative complications and/ or hospital LoS. Immunonutrition reduced total infective complications by $37 \%$, while IMT and exercise reduced PPCs by $45 \%$ and $46 \%$, respectively. LoS was reduced by 1.5-2 days on average.

Generally, these results were robust to sensitivity analyses. For immunonutrition, the results were attenuated after removing the studies at high $\mathrm{RoB}(\mathrm{LoS})$ and studies conducted prior to 2010 (total infective complications) (see online supplemental material). The overall quality of the evidence was low. For most interventions, overall pooled sample sizes were small and CIs were wide, suggesting that many of the analyses were underpowered. Overall, prehabilitation interventions appear safe; none of the studies reported adverse effects of any of the interventions administered and most studies achieved good follow-up and reported little or no attrition.

We found no evidence of subgroup effects by type of surgery. Despite the clinical heterogeneity in the studies identified (different surgical populations and differences between some interventions), statistical heterogeneity

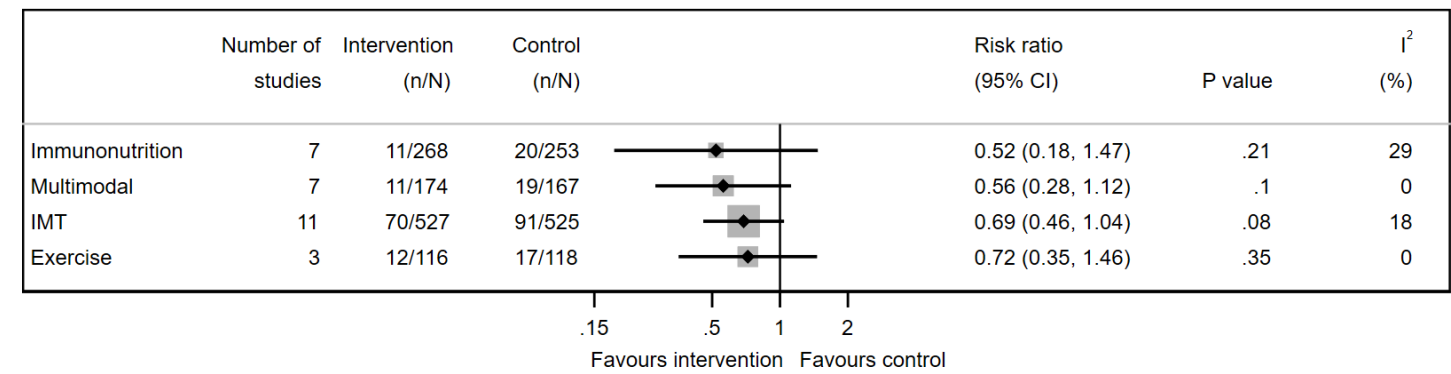

Figure 6 Forest plot of pre-admission interventions for reducing pneumonia. All interventions were tested with usual care as control. IMT, inspiratory muscle training. 
for most interventions and outcomes analysed was low, suggesting that our approach of pooling data across all surgical populations was justified. This is not surprising, given that the modifiable risk factors that reduce fitness for surgery and delay recovery are similar in different surgical populations. These risk factors include, for example, physical inactivity and low aerobic fitness, which affects between $33 \%$ and $45 \%$ of surgical populations, ${ }^{210}$ excessive alcohol consumption and smoking, which affects about a quarter, ${ }^{211}$ and obesity, which affects one-third. $^{212}$

There was marked clinical heterogeneity for some interventions, with variations in their components, duration and mode of administration; for example, educational interventions ranged from written information (a booklet) that patients were sent home with to one-to-one structured education programmes. Similarly, psychological interventions ranged from written instructions on relaxation exercises and deep breathing to cognitivebehavioural therapy sessions with a psychologist.

\section{Limitations of the evidence}

The trials that contributed data to these meta-analyses were largely of low or very low quality. Most trials were small (IMT 20-200 participants; immunonutrition 14-244 participants; exercise 14-164 participants) and not blinded, since blinding in trials of lifestyle interventions is challenging and often impossible. A systematic review of 45 trials reported that higher expectations of recovery positively influenced patient outcomes. ${ }^{213}$ Thus, the apparent intervention effect may be influenced by patient expectations rather than the active intervention. Nevertheless, IMT, exercise and immunonutrition trials all improved physiological markers of either exercise capacity (eg, inspiratory muscle strength, 6-minute walk test) or biomarkers of immune function, and these parameters remained unchanged in the usual care groups, which suggests that the interventions had physiological effects. The influence of blinding on effect size in trials is not clear cut; for example, a recent metaepidemiological study in physical therapy trials found no relationship between lack of blinding and effect size, and, surprisingly, trials with inadequate blinding tended to underestimate treatment effects. ${ }^{214} \mathrm{~A}$ further limitation for the immunonutrition trials was that they were mostly industry sponsored and there is evidence that industrysponsored studies are biased in favour of the sponsor's products. $^{209}$

Another limitation is the lack of standardised definitions of postoperative complications (infective, non-infective and pulmonary complications). Many studies reported individual complications without reporting total complications; therefore, we could not include them in metaanalyses. Similarly, LoS data were inconsistently reported (mean and SD or median and range or median and IQR) and in some cases no variance data were reported, so these studies could not be included in the meta-analysis. LoS is one of the main clinical outcomes of interest in this research area; however, it is likely to be influenced by variation in discharge criteria, which may result in differences between studies.

We had no information on whether studies were conducted pre-ERAS or post-ERAS implementation, and there is some evidence that beneficial effects of interventions carried out before surgery disappear when ERAS is introduced. ${ }^{215}$ However, our sensitivity analysis restricting to the previous 10 years (2010-2020), when most ERAS programmes were introduced, did not change effect sizes for any of the interventions and outcomes investigated.

Timing of mortality assessment within hospitals was not always clearly defined or consistent between studies. This lack of uniformity is likely to have caused rates of reporting to differ between studies. Further variability may have been introduced as definitions of some of our secondary outcomes of interest were not always clear and often differed between studies. An adequate description of the comparator (largely usual care) was absent from most study reports; it was therefore difficult to determine what usual care was, whether there were any enhanced recovery protocols in place, or even whether components were added to usual care for the purpose of the trial.

Few of the studies we identified reported outcomes beyond 30 days, therefore the effect of prehabilitation on longer term outcomes such as hospital readmission and mortality are not known. Also, the extent to which behaviour change in the preoperative period was maintained postoperatively, or whether this behaviour change leads to change in modifiable risk factors, remains to be assessed. Few studies (apart from the psychological intervention studies) included patient-reported outcomes such as QoL, pain, satisfaction with pre-admission intervention and care and factors associated with mental health. Finally, all interventions were initiated and followed up in hospital, with little consideration for how primary healthcare services could be integrated into the patient pathways for continuity of care after surgery.

\section{Strengths and limitations of the review}

A major strength of the review is that it provides a summary of the collective evidence on prehabilitation for all surgical patients. The systematic methods employed to identify the included studies were stringent, with inclusion of published literature in all languages, alongside grey literature searching, to avoid publication bias.

A limitation is that by having a stringent definition of prehabilitation as an intervention occurring only in the preoperative period, we excluded a lot of studies where the intervention continued postoperatively. This excluded 5 smoking cessation studies, in which the smoking cessation intervention almost always continues postoperatively, as well as 18 nutrition, 4 exercise, 6 respiratory, 1 multimodal, 3 education, 1 psychological, and 3 pharmacological intervention studies. A final limitation is the potential for effect size multiplicity ${ }^{216}$ (multiple dependent effect sizes, for example, in related outcomes such as infective complications, wound infection and pneumonia, derived 
from the same individual participants). We chose to perform separate analyses for each of these outcomes rather than averaging multiple effect sizes within studies because this is what we prespecified in our protocol and because we did not consider effect sizes for each outcome to be equivalent. We acknowledge the risk of inflating type 1 error rates.

\section{Deviations from the protocol}

We could not complete two of the subgroup analyses that we had prespecified (by differences in intervention characteristics and high vs low surgical risk patients) because of a lack of data. We also decided not to attempt to compare the effectiveness of the different components given the large variability within each intervention (in mode/place of administration, intensity and duration of intervention, etc).

\section{Agreements and disagreements with other research}

Despite the broad scope of this review, the inclusion of multiple surgical populations and the exclusion of studies in which the interventions were continued postoperatively, our results are similar to those reported for the specific prehabilitation interventions in single surgical populations.

\section{Future research/clinical recommendations}

Our review has highlighted that IMT, exercise, immunonutrition and smoking cessation interventions should be considered as part of multimodal prehabilitation programmes. Further research is needed on how best to identify the high-risk patients who are most likely to benefit from the various components of a multimodal prehabilitation intervention because treatment needs to be individualised, taking into account patient need, preferences and likelihood of adherence to the different components. For example, IMT could be an adjunct to exercise or replace exercise in those with impaired respiratory function, while a psychological intervention should only be offered to those who are likely to benefit, while a nutritional intervention needs to be tailored to whether the patient is malnourished or underweight or overweight. A well-designed, large, pragmatic, multicentre clinical trial is needed to determine the true effectiveness of an individualised multimodal intervention. Such a trial should collect long-term outcome data and patientreported outcomes data, including outcomes related to mental health. It should also measure adherence to the different components of the intervention and longer term behavioural/lifestyle changes. It should also investigate the mechanisms through which the different components of prehabilitation work. The impact of shorter LoS on the broader health and social care system and on longterm patient outcomes should also be considered. Future trials should also employ digital technology to monitor adherence and provide feedback to patients and also include aspects of implementation and scaling up of the interventions in the National Health Service (NHS).

\section{CONCLUSIONS}

Some prehabilitation interventions, in particular IMT and immunonutrition, may reduce hospital LoS and some postoperative complications. Overall, the quality of the evidence was low or very low. Despite the relatively large number of studies identified, most had very small sample sizes and our pooled analyses were likely underpowered.

\section{Author affiliations}

${ }^{1} \mathrm{NIHR}$ Bristol BRC, University Hospitals Bristol and Weston NHS Foundation Trust,

University of Bristol, Bristol, UK

${ }^{2}$ Centre for Exercise, Nutrition and Health Sciences, University of Bristol, Bristol, UK ${ }^{3}$ Population Health Sciences, Bristol Medical School, University of Bristol, Bristol, UK ${ }^{4}$ Bristol Trials Centre (CTEU), Bristol Medical School, University of Bristol, Bristol, UK ${ }^{5}$ School of Oral and Dental Science, University of Bristol, Bristol, UK

${ }^{6}$ Faculty of Health, Victoria University of Wellington, Wellington, New Zealand ${ }^{7}$ University Hospitals Bristol and Weston NHS Foundation Trust, University of Bristol, Bristol, UK

Acknowledgements We thank Alison Richards and Sarah Dawson for conducting the literature searches, Zoe Zou and Sarah Sauchelli Toran for translating texts into English, Shirley Jenkins and Sofia Leadbetter for help with referencing and Alex Whitmarsh for statistical advice. The following contributed to abstract screening or data extraction: Sharea ljaz, Lauren Scott, Agnieszka Skorko, George Snell, Helena Wilkes, Mariella Williams and Annabelle Johnson. We also thank Professor Julian Higgins for general advice on systematic review conduct and reporting.

Contributors RP conducted the study (screened abstracts and full texts, extracted data, assessed risk of bias, conducted GRADE assessmet, conducted all analyses and assembled tables of results) and wrote the first draft of the manuscript. GH conducted the study (extracted data, assessed risk of bias, completed handsearching, assembled tables of results, checked analysis, conducted GRADE assessment). CA and CE conducted the study (extracted data, completed handsearching and assembled tables of results, checked analysis and formatting, conducted GRADE assessment). KN, SB, TB, AC and AH extracted data. JH conducted some of the statistical analyses (Egger's regression tests) and constructed all the plots in the main manuscript. AN critically reviewed the manuscript. SS had the idea for conducting a study of prehabilitation in patients undergoing major elective surgery and provided clinical input. MP designed and conducted the study, interpreted the data and wrote the manuscript. The manuscript's guarantors (MP and RP) affirm that the manuscript is an honest, accurate, and transparent account of the study being reported; that no important aspects of the study have been omitted; and that any discrepancies from the study as planned (and, if relevant, registered) have been explained.

Funding This work was supported by the Elizabeth Blackwell Institute (University of Bristol) and the Bristol National Institutes of Health Research (NIHR) Biomedical Research Centre.

Competing interests None declared.

Patient consent for publication Not applicable.

Provenance and peer review Not commissioned; externally peer reviewed.

Data availability statement Data are available upon reasonable request. "The aggregate data for all the meta-analyses that were conducted are listed in in the Supplementary Digital Content. These data were entered into RevMan and can be made available as an XML file upon request from the corresponding author.".

Supplemental material This content has been supplied by the author(s). It has not been vetted by BMJ Publishing Group Limited (BMJ) and may not have been peer-reviewed. Any opinions or recommendations discussed are solely those of the author(s) and are not endorsed by BMJ. BMJ disclaims all liability and responsibility arising from any reliance placed on the content. Where the content includes any translated material, BMJ does not warrant the accuracy and reliability of the translations (including but not limited to local regulations, clinical guidelines, terminology, drug names and drug dosages), and is not responsible for any error and/or omissions arising from translation and adaptation or otherwise.

Open access This is an open access article distributed in accordance with the Creative Commons Attribution 4.0 Unported (CC BY 4.0) license, which permits others to copy, redistribute, remix, transform and build upon this work for any purpose, provided the original work is properly cited, a link to the licence is given, 
and indication of whether changes were made. See: https://creativecommons.org/ licenses/by/4.0/.

ORCID iD

Rachel Perry http://orcid.org/0000-0001-5874-3016

\section{REFERENCES}

1 Abbott TEF, Fowler AJ, Dobbs TD, et al. Frequency of surgical treatment and related Hospital procedures in the UK: a national ecological study using Hospital episode statistics. $\mathrm{Br} J$ Anaesth 2017;119:249-57.

2 Grocott MPW, Browne JP, Van der Meulen J, et al. The postoperative morbidity survey was validated and used to describe morbidity after major surgery. J Clin Epidemiol 2007;60:919-28.

3 Lai CW, Minto G, Challand CP, et al. Patients' inability to perform a preoperative cardiopulmonary exercise test or demonstrate an anaerobic threshold is associated with inferior outcomes after major colorectal surgery. Br J Anaesth 2013;111:607-11.

4 Snowden CP, Prentis JM, Anderson HL, et al. Submaximal cardiopulmonary exercise testing predicts complications and hospital length of stay in patients undergoing major elective surgery. Ann Surg 2010;251:535-41.

5 Snowden CP, Prentis J, Jacques B, et al. Cardiorespiratory fitness predicts mortality and hospital length of stay after major elective surgery in older people. Ann Surg 2013;257:999-1004.

6 Kehlet H, Wilmore DW. Evidence-Based surgical care and the evolution of fast-track surgery. Ann Surg 2008;248:189-98.

7 O'Doherty AF, West M, Jack S, et al. Preoperative aerobic exercise training in elective intra-cavity surgery: a systematic review. $\mathrm{Br} J$ Anaesth 2013;110:679-89.

8 Santa Mina D, Clarke H, Ritvo P, et al. Effect of total-body prehabilitation on postoperative outcomes: a systematic review and meta-analysis. Physiotherapy 2014;100:196-207.

9 Hulzebos EHJ, Smit Y, Helders PPJM, et al. Preoperative physical therapy for elective cardiac surgery patients. Cochrane Database Syst Rev 2012;11:Cd010118.

10 Lemanu DP, Singh PP, MacCormick AD, et al. Effect of preoperative exercise on cardiorespiratory function and recovery after surgery: a systematic review. World J Surg 2013;37:711-20.

11 Piraux E, Caty G, Reychler G. Effects of preoperative combined aerobic and resistance exercise training in cancer patients undergoing tumour resection surgery: a systematic review of randomised trials. Surg Oncol 2018;27:584-94.

12 Pouwels S, Stokmans RA, Willigendael EM, et al. Preoperative exercise therapy for elective major abdominal surgery: a systematic review. Int J Surg 2014;12:134-40.

13 Singh F, Newton RU, Galvão DA, et al. A systematic review of presurgical exercise intervention studies with cancer patients. Surg Oncol 2013;22:92-104.

14 Burden S, Todd C, Hill J, et al. Pre-Operative nutrition support in patients undergoing gastrointestinal surgery. Cochrane Database Syst Rev 2012;11:Cd008879.

15 Thomsen T, Villebro N, Møller AM. Interventions for preoperative smoking cessation. Cochrane Database Syst Rev 2014;2014:Cd002294.

16 Oppedal K, Møller AM, Pedersen B, et al. Preoperative alcohol cessation prior to elective surgery. Cochrane Database Syst Rev 2012;7:Cd008343.

17 Katsura M, Kuriyama A, Takeshima T, et al. Preoperative inspiratory muscle training for postoperative pulmonary complications in adults undergoing cardiac and major abdominal surgery. Cochrane Database Syst Rev 2015;10:Cd010356.

18 McDonald S, Page MJ, Beringer K. Preoperative education for hip or knee replacement. Cochrane Database Syst Rev 2014;2014:Cd003526.

19 Li C, Carli F, Lee L, et al. Impact of a trimodal prehabilitation program on functional recovery after colorectal cancer surgery: a pilot study. Surg Endosc 2013;27:1072-82.

20 Jack S, West M, Grocott MPW. Perioperative exercise training in elderly subjects. Best Pract Res Clin Anaesthesiol 2011;25:461-72.

21 Steffens D, Beckenkamp PR, Hancock M, et al. Preoperative exercise halves the postoperative complication rate in patients with lung cancer: a systematic review of the effect of exercise on complications, length of stay and quality of life in patients with cancer. Br J Sports Med 2018;52:344.

22 Thybo Karanfil EO, Møller AM. Preoperative inspiratory muscle training prevents pulmonary complications after cardiac surgery - a systematic review. Dan Med J 2018;65.
23 Howes N, Atkinson C, Thomas S, et al. Immunonutrition for patients undergoing surgery for head and neck cancer. Cochrane Database Syst Rev 2018;8:Cd010954.

24 Perry R, Scott LJ, Richards A, et al. Pre-Admission interventions to improve outcome after elective surgery-protocol for a systematic review. Syst Rev 2016;5:88.

25 Higgins J, Green S. Cochrane Handbook for Systematic Reviews of Interventions Version 5.1.0 [updated March 2011]. The Cochrane Collaboration, 2011.

26 Guyatt GH, Oxman AD, Vist GE, et al. Grade: an emerging consensus on rating quality of evidence and strength of recommendations. BMJ 2008;336:924-6.

27 McGrath S, Zhao X, Steele R, et al. Estimating the sample mean and standard deviation from commonly reported quantiles in meta-analysis. Stat Methods Med Res 2020:962280219889080 (published Online First: 2020/04/16).

28 Cortés J, González JA, Campbell MJ, et al. A hazard ratio was estimated by a ratio of median survival times, but with considerable uncertainty. J Clin Epidemiol 2014;67:1172-7.

29 Deeks JJ, Higgins JPT, Altman DG. Chapter 9: Analysing data and undertaking meta-analyses. In: Higgins JPT, Green S, eds. Cochrane Handbook for systematic reviews of interventions version 5.1.0. The Cochrane Collaboration, 2011. www.cochranehandbook.org

30 Burden ST, Hill J, Shaffer JL, et al. An unblinded randomised controlled trial of preoperative oral supplements in colorectal cancer patients. J Hum Nutr Diet 2011;24:441-8.

31 Burden ST, Gibson DJ, Lal S, et al. Pre-Operative oral nutritional supplementation with dietary advice versus dietary advice alone in weight-losing patients with colorectal cancer: single-blind randomized controlled trial. J Cachexia Sarcopenia Muscle 2017;8:437-46.

32 Kikuchi Y, Hiroshima Y, Matsuo K, et al. A randomized clinical trial of preoperative administration of branched-chain amino acids to prevent postoperative ascites in patients with liver resection for hepatocellular carcinoma. Ann Surg Oncol 2016;23:3727-35.

33 MacFie J, Woodcock NP, Palmer MD, et al. Oral dietary supplements in pre- and postoperative surgical patients: a prospective and randomized clinical trial. Nutrition 2000;16:723-8.

34 Nagata S, Shirabe K, Sugimachi K, et al. Pilot study of preoperative immunonutrition with antioxidants in living donor liver transplantation donors. Fukuoka Igaku Zasshi 2013;104:530-8.

35 Smedley F, Bowling T, James M, et al. Randomized clinical trial of the effects of preoperative and postoperative oral nutritional supplements on clinical course and cost of care. Br J Surg 2004;91:983-90.

36 Aida T, Furukawa K, Suzuki D, et al. Preoperative immunonutrition decreases postoperative complications by modulating prostaglandin E2 production and T-cell differentiation in patients undergoing pancreatoduodenectomy. Surgery 2014;155:124-33.

37 Barker LA, Gray C, Wilson L, et al. Preoperative immunonutrition and its effect on postoperative outcomes in well-nourished and malnourished gastrointestinal surgery patients: a randomised controlled trial. Eur J Clin Nutr 2013;67:802-7.

38 Braga M, Gianotti L, Vignali A, et al. Preoperative oral arginine and $\mathrm{n}-3$ fatty acid supplementation improves the immunometabolic host response and outcome after colorectal resection for cancer. Surgery 2002;132:805-14.

39 Fujitani K, Tsujinaka T, Fujita J, et al. Prospective randomized trial of preoperative enteral immunonutrition followed by elective total gastrectomy for gastric cancer. Br J Surg 2012;99:621-9.

40 Gianotti L, Braga M, Nespoli L, et al. A randomized controlled trial of preoperative oral supplementation with a specialized diet in patients with gastrointestinal cancer. Gastroenterology 2002;122:1763-70.

41 Gunerhan Y, Koksal N, Sahin UY, et al. Effect of preoperative immunonutrition and other nutrition models on cellular immune parameters. World J Gastroenterol 2009;15:467-72.

42 Kaya SO, Akcam TI, Ceylan KC, et al. Is preoperative protein-rich nutrition effective on postoperative outcome in non-small cell lung cancer surgery? A prospective randomized study. J Cardiothorac Surg 2016;11:14.

43 Manzanares Campillo MDC, Martín Fernández J, Amo Salas $\mathrm{M}$, et al. [A randomized controlled trial of preoperative oral immunonutrition in patients undergoing surgery for colorectal cancer: hospital stay and health care costs]. Cir Cir 2017;85:393-400.

44 Mikagi K, Kawahara R, Kinoshita H, et al. Effect of preoperative immunonutrition in patients undergoing hepatectomy; a randomized controlled trial. Kurume Med J 2011;58:1-8.

45 Nakamura K, Kariyazono H, Komokata T, et al. Influence of preoperative administration of omega-3 fatty acid-enriched 
supplement on inflammatory and immune responses in patients undergoing major surgery for cancer. Nutrition 2005;21:639-49.

46 Pronio A, Di Filippo A, Aguzzi D, et al. [Treatment of mild malnutrition and reduction of morbidity in major abdominal surgery: randomized trial on 153 patients]. Clin Ter 2008;159:13-18.

47 Sufit A, Weitzel LB, Hamiel C, et al. Pharmacologically dosed oral glutamine reduces myocardial injury in patients undergoing cardiac surgery: a randomized pilot feasibility trial. JPEN J Parenter Enteral Nutr 2012;36:556-61.

48 Alami RS, Morton JM, Schuster R, et al. Is there a benefit to preoperative weight loss in gastric bypass patients? A prospective randomized trial. Surg Obes Relat Dis 2007;3:141-5. discussion 45-6.

49 Barth RJ, Mills JB, Suriawinata AA, et al. Short-Term preoperative diet decreases bleeding after partial hepatectomy: results from a multi-institutional randomized controlled trial. Ann Surg 2019;269:48-52.

50 Bottin J, Balogun B, Thomas E. Changes in body composition induced by pre-operative liquid low-calorie diet in morbid obese patients undergoing Roux-en-Y gastric bypass. Obesity Reviews 2014;15:129-30.

51 Chakravartty S, Vivian G, Mullholland N, et al. Preoperative liver shrinking diet for bariatric surgery may impact wound healing: a randomized controlled trial. Surg Obes Relat Dis 2019;15:117-25.

52 Elrefai M. Value of low calorie diet before sleeve gastrectomy: Prospectieve randomised study. pre-operative management. Obesity Surgery 2017;27:813.

53 Faria SL, Faria OP, de Almeida Cardeal M, et al. Effects of a very low calorie diet in the preoperative stage of bariatric surgery: a randomized trial. Surg Obes Relat Dis 2015;11:230-7.

54 Grundmann F, Müller R-U, Reppenhorst A, et al. Preoperative shortterm calorie restriction for prevention of acute kidney injury after cardiac surgery: a randomized, controlled, open-label, pilot trial. J Am Heart Assoc 2018;7. doi:10.1161/JAHA.117.008181. [Epub ahead of print: 13032018 .

55 Schouten R, van der Kaaden I, van 't Hof G, et al. Comparison of preoperative diets before bariatric surgery: a randomized, singleblinded, non-inferiority trial. Obes Surg 2016;26:1743-9.

56 van Ginhoven TM, de Bruin RWF, Timmermans M, et al. PreOperative dietary restriction is feasible in live-kidney donors. Clin Transplant 2011;25:486-94.

57 Van Nieuwenhove Y, Dambrauskas Z, Campillo-Soto A, et al. Preoperative very low-calorie diet and operative outcome after laparoscopic gastric bypass: a randomized multicenter study. Arch Surg 2011;146:1300-5.

58 Anderson ADG, McNaught CE, Jain PK, et al. Randomised clinical trial of synbiotic therapy in elective surgical patients. Gut 2004;53:241-5

59 Consoli MLD, da Silva RS, Nicoli JR, et al. Randomized clinical trial: impact of oral administration of Saccharomyces boulardii on gene expression of intestinal cytokines in patients undergoing colon resection. JPEN J Parenter Enteral Nutr 2016:40:1114-21.

60 Grąt M, Wronka KM, Lewandowski Z, et al. Effects of continuous use of probiotics before liver transplantation: a randomized, doubleblind, placebo-controlled trial. Clin Nutr 2017;36:1530-9.

61 Krebs B. Prebiotic and Synbiotic Treatment before Colorectal Surgery--Randomised Double Blind Trial. Coll Antropol 2016;40:35-40.

62 Chuah L, Miras A, Noon J. Does intensive preoperative and postoperative glucose management influence glycaemic outcome of Roux-en-Y gastric bypass surgery? Obesity Reviews 2014;15:136

63 Flynn MB, Leightty FF. Preoperative outpatient nutritional support of patients with squamous cancer of the upper aerodigestive tract. $A m$ J Surg 1987:154:359-62.

64 Patel VC, Aldridge RD, Leeds A, et al. Retrospective analysis of the impact of a low glycaemic index diet on hospital stay following coronary artery bypass grafting: a hypothesis. J Hum Nutr Diet 2004;17:241-7.

65 Akbarzadeh M, Eftekhari MH, Shafa M, et al. Effects of a new metabolic conditioning supplement on perioperative metabolic stress and clinical outcomes: a randomized, placebo-controlled trial. Iran Red Crescent Med J 2016;18:e26207.

66 Makhija N, Sendasgupta C, Kiran U, et al. The role of oral coenzyme Q10 in patients undergoing coronary artery bypass graft surgery. $J$ Cardiothorac Vasc Anesth 2008;22:832-9.

67 Matzi V, Lindenmann J, Muench A, et al. The impact of preoperative micronutrient supplementation in lung surgery. A prospective randomized trial of oral supplementation of combined alphaketoglutaric acid and 5-hydroxymethylfurfural. Eur J Cardiothorac Surg 2007;32:776-82.
68 Metcalf RG, James MJ, Gibson RA, et al. Effects of fish-oil supplementation on myocardial fatty acids in humans. Am J Clin Nutr 2007;85:1222-8.

69 Zhao Q, Li Y, Yu B, et al. Effects of preoperative enteral nutrition on postoperative recent nutritional status in patients with Siewert II and III adenocarcinoma of esophagogastric junction after neoadjuvant chemoradiotherapy. Nutr Cancer 2018;70:895-903.

70 Desai S, Vijayashree N, Merina A. Effect of preoperative L-glutamine supplementation on primary outcomes in elective cardiac surgery. Eur Heart J 2016;37:729.

71 Hossain LE, Gonzalez FG, Manuel RR. Effect of "immunonutrition" compared to standard nutrition in gastrointestinal malignancies. Surgical Infections 2016;17:S27.

72 Martinez JL, Bosco-Garate I, Souza-Gallardo LM, et al. Effect of preoperative administration of oral arginine and glutamine in patients with enterocutaneous fistula submitted to definitive surgery: a prospective randomized trial. J Gastrointest Surg 2020;24:426-34.

73 Russell K, Zhang H-G, Gillanders LK, et al. Preoperative immunonutrition in patients undergoing liver resection: a prospective randomized trial. World J Hepatol 2019;11:305-17.

74 Tumas J, Jasiunas E, Strupas K. Effects of immunonutrition on comprehensive complication index in patients undergoing pancreatoduodenectomy. Medicina-Lithuania 2020;56.

75 Yoshitomi M, Kawahara R, Akasu G. Preoperative immunonutrition for patients undergoing pancreatoduodenectomy (randomized controlled trial). Pancreas 2009;38:1066.

76 Hollis G, Franz R, Bauer J, et al. Implementation of a very low calorie diet program into the pre-operative model of care for obese General elective surgery patients: outcomes of a feasibility randomised control trial. Nutr Diet 2020;77:490-498.

77 Polakowski CB, Kato M, Preti VB, et al. Impact of the preoperative use of synbiotics in colorectal cancer patients: a prospective, randomized, double-blind, placebo-controlled study. Nutrition 2019;58:40-6.

78 Zhang J-W, Du P, Gao J, et al. Preoperative probiotics decrease postoperative infectious complications of colorectal cancer. $A m \mathrm{~J}$ Med Sci 2012;343:199-205.

79 Krasowska K, Skrobot W, Liedtke E, et al. The preoperative supplementation with vitamin $\mathrm{D}$ attenuated pain intensity and reduced the level of pro-inflammatory markers in patients after posterior lumbar interbody fusion. Front Pharmacol 2019;10:527.

80 Gade J, Levring T, Hillings $\varnothing \mathrm{J}$, et al. The Effect of Preoperative Oral Immunonutrition on Complications and Length of Hospital Stay After Elective Surgery for Pancreatic Cancer--A Randomized Controlled Trial. Nutr Cancer 2016;68:225-33.

81 Barbalho-Moulim MC, Miguel GPS, Forti EMP, et al. Effects of preoperative inspiratory muscle training in obese women undergoing open bariatric surgery: respiratory muscle strength, lung volumes, and diaphragmatic excursion. Clinics 2011;66:1721-7.

82 Carvalho T, Bonorino KC, Panigas TF. Preoperative respiratory muscle training reduces complications in coronary artery bypass surgery [abstract]. Eur Heart J2011;32.

83 Da Cunha F, Ruas G, Fanan J. Effects of preoperative respiratory muscle training on early and late postoperative outcome of patients undergoing esophageal surgery. Intensive Care Medicine 2013;39:S369.

84 Dronkers J, Veldman A, Hoberg E, et al. Prevention of pulmonary complications after upper abdominal surgery by preoperative intensive inspiratory muscle training: a randomized controlled pilot study. Clin Rehabil 2008:22:134-42.

85 Dronkers JJ, Lamberts H, Reutelingsperger IMMD, et al. Preoperative therapeutic programme for elderly patients scheduled for elective abdominal oncological surgery: a randomized controlled pilot study. Clin Rehabil 2010;24:614-22.

86 Ferreira PEG, Rodrigues AJ, Evora PRB. Effects of an inspiratory muscle rehabilitation program in the postoperative period of cardiac surgery. Arq Bras Cardiol 2009;92:275-82.

87 Hulzebos EHJ, Helders PJM, Favié NJ, et al. Preoperative intensive inspiratory muscle training to prevent postoperative pulmonary complications in high-risk patients undergoing CABG surgery: a randomized clinical trial. JAMA 2006;296:1851-7.

88 Hulzebos EHJ, van Meeteren NLU, van den Buijs BJWM, et al. Feasibility of preoperative inspiratory muscle training in patients undergoing coronary artery bypass surgery with a high risk of postoperative pulmonary complications: a randomized controlled pilot study. Clin Rehabil 2006;20:949-59.

89 Jarosz A, Szlubowski A, Grochowski Z, et al. P-120 * the evaluation of utility of preoperative Systematized PULMONOLOGICAL physiotherapy among non-small-cell lung cancer patients 
undergoing anatomical lung resection. Interact Cardiovasc Thorac Surg 2014;18:S32.

90 Kulkarni SR, Fletcher E, McConnell AK, et al. Pre-operative inspiratory muscle training preserves postoperative inspiratory muscle strength following major abdominal surgery - a randomised pilot study. Ann R Coll Surg Engl 2010;92:700-7.

$91 \mathrm{Ma} \mathrm{B}, \mathrm{Bao} \mathrm{H}$. Reduction in pulmonary complications in high risk patients undergoing surgery for total hip replacement under general anesthesia by preoperative intensive inspiratory muscle training: A randomized controlled clinical trial. Journal of Nanjing Medical University 2009;23:328-34.

92 Sobrinho MT, Guirado GN, Silva MAdeM. Preoperative therapy restores ventilatory parameters and reduces length of stay in patients undergoing myocardial revascularization. Rev Bras Cir Cardiovasc 2014;29:221-8.

93 Valkenet K, Trappenburg JCA, Ruurda JP, et al. Multicentre randomized clinical trial of inspiratory muscle training versus usual care before surgery for oesophageal cancer. Br J Surg 2018; 105:502-11.

94 van den Buijs BJW, Hulzebos HJ, Bie R. Preoperative inspiratory muscle training in patients due to undergo open-heart surgery: a pilot study. Dutch J Phys Ther 2004;114:104-9.

95 Weiner P, Zeidan F, Zamir D, et al. Prophylactic inspiratory muscle training in patients undergoing coronary artery bypass graft. World J Surg 1998;22:427-31.

96 Bergin C, Speroni KG, Travis T, et al. Effect of preoperative incentive spirometry patient education on patient outcomes in the knee and hip joint replacement population. J Perianesth Nurs 2014;29:20-7.

97 Celli BR, Rodriguez KS, Snider GL. A controlled trial of intermittent positive pressure breathing, incentive spirometry, and deep breathing exercises in preventing pulmonary complications after abdominal surgery. Am Rev Respir Dis 1984;130:12-15.

98 Cattano D, Altamirano A, Vannucci A, et al. Preoperative use of incentive spirometry does not affect postoperative lung function in bariatric surgery. Trans/ Res 2010;156:265-72.

99 Leguisamo CP, Kalil RAK, Furlani AP. A efetividade de uma proposta fisioterapêutica pré-operatória para cirurgia de revascularização do miocárdio. Brazilian Journal of Cardiovascular Surgery 2005;20:134-41.

100 Benzo R, Wigle D, Novotny P, et al. Preoperative pulmonary rehabilitation before lung cancer resection: results from two randomized studies. Lung Cancer 2011;74:441-5.

101 Lloréns J, Rovira L, Ballester M, et al. Preoperative inspiratory muscular training to prevent postoperative hypoxemia in morbidly obese patients undergoing laparoscopic bariatric surgery. A randomized clinical trial. Obes Surg 2015;25:1003-9.

102 Ortega J, Cassinello N, Rovira L. Preoperative respiratory physiotherapy can improve oxygenation during bariatric surgery 2013.

103 Rajendran AJ, Pandurangi UM, Murali R, et al. Pre-Operative shortterm pulmonary rehabilitation for patients of chronic obstructive pulmonary disease undergoing coronary artery bypass graft surgery. Indian Heart J 1998;50:531-4.

104 Moreno J, Rovira L, Hernandez J. Effect of preoperatory chest physiotherapy program on the oxigenation, after laparoscopic bariatric surgery: 5AP3-8. European Journal of Anaesthesiology 2012;29:88.

105 Yamana I, Takeno S, Hashimoto T, et al. Randomized controlled study to evaluate the efficacy of a preoperative respiratory rehabilitation program to prevent postoperative pulmonary complications after esophagectomy. Dig Surg 2015;32:331-7.

106 Chen X, Hou L, Zhang Y, et al. The effects of five days of intensive preoperative inspiratory muscle training on postoperative complications and outcome in patients having cardiac surgery: a randomized controlled trial. Clin Rehabil 2019;33:913-22.

107 Devecel G, Senturan L. The effect of breathing exercises on preventing postoperative atelectasis: a randomized controlled trial. Florence Nightingale Hemşirelik Dergisi 2018;26:159-67.

108 Huang J, Lai Y, Zhou X, et al. Short-Term high-intensity rehabilitation in radically treated lung cancer: a three-armed randomized controlled trial. J Thorac Dis 2017;9:1919-29.

109 Laurent H, Aubreton S, Galvaing G, et al. Preoperative respiratory muscle endurance training improves ventilatory capacity and prevents pulmonary postoperative complications after lung surgery. Eur J Phys Rehabil Med 2020;56:73-81.

110 Argunova YA, Korotkevich AA, Pomeshkina SA, et al. Efficacy of physical trainings as cardioprotection method for coronary bypass surgery. Russian Journal of Cardiology 2018;23:159-65.

111 Banerjee S, Manley K, Shaw B, et al. Vigorous intensity aerobic interval exercise in bladder cancer patients prior to radical cystectomy: a feasibility randomised controlled trial. Support Care Cancer 2018;26:1515-23.

112 Barakat HM, Shahin Y, Khan JA, et al. Preoperative supervised exercise improves outcomes after elective abdominal aortic aneurysm repair: a randomized controlled trial. Ann Surg 2016;264:47-53.

113 Bridevaux P-O, Tschopp J-M, Bhatia C. Effect of pre-operative short-term rehabilitation on peak VO2 in patients with NSCLC. Eur Res J 2012;40:3306.

114 Carver TE, Mayo N, Andersen RE, et al. Pilot investigation to evaluate changes in exercise capacity following a prehabilitation intervention among seriously obese patients awaiting bariatric surgery. Can J Diabetes 2011;35:149.

115 Cavill S, McKenzie K, Munro A, et al. The effect of prehabilitation on the range of motion and functional outcomes in patients following the total knee or hip arthroplasty: a pilot randomized trial. Physiother Theory Pract 2016;32:262-70.

116 D'Lima DD, Colwell CW, Morris BA, et al. The effect of preoperative exercise on total knee replacement outcomes. Clin Orthop Relat Res 1996:174-82.

117 Dunne DFJ, Jack S, Jones RP, et al. Randomized clinical trial of prehabilitation before planned liver resection. Br J Surg 2016;103:504-12

118 Sebio García R, Yáñez-Brage MI, Giménez Moolhuyzen E, et al. Preoperative exercise training prevents functional decline after lung resection surgery: a randomized, single-blind controlled trial. Clin Rehabil 2017;31:1057-67.

119 Hoogeboom TJ, Dronkers JJ, van den Ende CHM, et al. Preoperative therapeutic exercise in frail elderly scheduled for total hip replacement: a randomized pilot trial. Clin Rehabil 2010;24:901-10.

120 Kaibori M, Ishizaki M, Matsui K, et al. Perioperative exercise for chronic liver injury patients with hepatocellular carcinoma undergoing hepatectomy. Am J Surg 2013;206:202-9.

121 Licker M, Karenovics W, Diaper J, et al. Short-Term Preoperative High-Intensity Interval Training in Patients Awaiting Lung Cancer Surgery: A Randomized Controlled Trial. J Thorac Oncol 2017;12:323-33.

122 Lindbäck Y, Tropp H, Enthoven P, et al. Prepare: presurgery physiotherapy for patients with degenerative lumbar spine disorder: a randomized controlled trial. Spine J 2018;18:1347-55.

123 Oosting E, Jans MP, Dronkers JJ, et al. Preoperative home-based physical therapy versus usual care to improve functional health of frail older adults scheduled for elective total hip arthroplasty: a pilot randomized controlled trial. Arch Phys Med Rehabil 2012;93:610-6.

124 Rooks DS, Huang J, Bierbaum BE, et al. Effect of preoperative exercise on measures of functional status in men and women undergoing total hip and knee arthroplasty. Arthritis Rheum 2006:55:700-8.

125 Santa Mina D, Hilton WJ, Matthew AG, et al. Prehabilitation for radical prostatectomy: a multicentre randomized controlled trial. Surg Oncol 2018;27:289-98.

126 Soares SMdeTP, Nucci LB, da Silva MMdeC, et al. Pulmonary function and physical performance outcomes with preoperative physical therapy in upper abdominal surgery: a randomized controlled trial. Clin Rehabil 2013;27:616-27.

127 Tenconi S, Galeone C, Fugazzaro S, et al. F-079PERIOPERATIVE and long-term effects of comprehensive pulmonary rehabilitation on exercise capacity, postoperative outcome and quality of life in patients undergoing lung resection: a randomized controlled trial granted by the Ministry of health. Interact Cardiovasc Thorac Surg 2017;25.

128 Tew GA, Batterham AM, Colling K, et al. Randomized feasibility trial of high-intensity interval training before elective abdominal aortic aneurysm repair. Br J Surg 2017;104:1791-801.

129 Wang AW, Gilbey HJ, Ackland TR. Perioperative exercise programs improve early return of ambulatory function after total hip arthroplasty: a randomized, controlled trial. Am J Phys Med Rehabil 2002;81:801-6.

130 Blackwell JEM, Doleman B, Boereboom CL, et al. High-Intensity interval training produces a significant improvement in fitness in less than 31 days before surgery for urological cancer: a randomised control trial. Prostate Cancer Prostatic Dis 2020;23:696-704.

131 Mak T, Futaba K, Leung WW. Multidisciplinary prehabilitation programme on patients undergoing colorectal cancer surgery: a single centre randomised controlled trial. Colorectal Disease 2019;21:16

132 Marchand A-A, Suitner M, O'Shaughnessy J, et al. Feasibility of conducting an active exercise prehabilitation program in patients awaiting spinal stenosis surgery: a randomized pilot study. Sci Rep 2019;9:12257. 
$133 \mathrm{McHugh}$ G. The role of perhabilitation on the outcome of total knee arthroplasty: a randomized control trial 2011.

134 Nolan F, Lyon K, Lambie N. Initial results: the effect of a physiotherapy prehabilitation programme on postoperative outcomes in patients undergoing cardiac or thoracic surgery. Clin Nutr ESPEN 2019;31:111.

135 Northgraves MJ, Arunachalam L, Madden LA, et al. Feasibility of a novel exercise prehabilitation programme in patients scheduled for elective colorectal surgery: a feasibility randomised controlled trial. Support Care Cancer 2020;28:3197-206.

136 Maguire S, Kinsella J, Steele R. Does prehabilitation modify muscle wasting in patients with rectal cancer undergoing neoadjuvant therapy? Colorectal Disease 2018;20:9-10.

137 Abdelaal GA, Eldahdouh SS, Abdelsamie M, et al. Effect of preoperative physical and respiratory therapy on postoperative pulmonary functions and complications after laparoscopic upper abdominal surgery in obese patients. Egypt $J$ Chest Dis Tuberc 2017;66:735-8.

138 Ausania F, Senra P, Meléndez R, et al. Prehabilitation in patients undergoing pancreaticoduodenectomy: a randomized controlled trial. Rev Esp Enferm Dig 2019;111:603-8.

139 Arthur HM, Daniels C, McKelvie R, et al. Effect of a preoperative intervention on preoperative and postoperative outcomes in low-risk patients awaiting elective coronary artery bypass graft surgery. A randomized, controlled trial. Ann Intern Med 2000;133:253-62.

140 Barberan-Garcia A, Ubré M, Roca J, et al. Personalised prehabilitation in high-risk patients undergoing elective major abdominal surgery: a randomized blinded controlled trial. Ann Surg 2018;267:50-6.

141 Beaupre LA, Lier D, Davies DM, et al. The effect of a preoperative exercise and education program on functional recovery, health related quality of life, and health service utilization following primary total knee arthroplasty. J Rheumatol 2004;31:1166-73.

142 Bousquet-Dion G, Awasthi R, Loiselle Sarah-Ève, Loiselle S, et al. Evaluation of supervised multimodal prehabilitation programme in cancer patients undergoing colorectal resection: a randomized control trial. Acta Oncol 2018;57:849-59.

143 Crowe J, Henderson J. Pre-arthroplasty rehabilitation is effective in reducing hospital stay. Can J Occup Ther 2003;70:88-96.

144 Demark-Wahnefried W, Nix JW, Hunter GR, et al. Feasibility outcomes of a presurgical randomized controlled trial exploring the impact of caloric restriction and increased physical activity versus a wait-list control on tumor characteristics and circulating biomarkers in men electing prostatectomy for prostate cancer. BMC Cancer 2016;16:61.

145 Huang S-W, Chen P-H, Chou Y-H. Effects of a preoperative simplified home rehabilitation education program on length of stay of total knee arthroplasty patients. Orthop Traumatol Surg Res 2012;98:259-64.

146 Kassouf W, Minnella E, Awasthi R. Prehabilitation for patients undergoing cystectomy: preliminary analysis of a single-center, randomized controlled trial. Journal of Urology2018;199:e622.

147 Kasvis P, Bui T, Kilgour R. A multimodal prehabilitation program in hepato-pancreato-biliary cancer patients awaiting surgery: preliminary results. MASCC/ISOO annual meeting on supportive care in cancer 2018.

148 Lai Y, Huang J, Yang M, et al. Seven-Day intensive preoperative rehabilitation for elderly patients with lung cancer: a randomized controlled trial. J Surg Res 2017;209:30-6.

149 Morano MT, Araújo AS, Nascimento FB, et al. Preoperative pulmonary rehabilitation versus chest physical therapy in patients undergoing lung cancer resection: a pilot randomized controlled trial. Arch Phys Med Rehabil 2013;94:53-8.

150 Ommundsen N, Wyller TB, Nesbakken A, et al. Preoperative geriatric assessment and tailored interventions in frail older patients with colorectal cancer: a randomized controlled trial. Colorectal Dis 2018;20:16-25

151 Pehlivan E, Turna A, Gurses A, et al. The effects of preoperative short-term intense physical therapy in lung cancer patients: a randomized controlled trial. Ann Thorac Cardiovasc Surg 2011;17:461-8

152 Rosenfeldt F, Braun L, Spitzer O, et al. Physical conditioning and mental stress reduction--a randomised trial in patients undergoing cardiac surgery. BMC Complement Altern Med 2011;11:20.

153 Sawatzky J-AV, Kehler DS, Ready AE, et al. Prehabilitation program for elective coronary artery bypass graft surgery patients: a pilot randomized controlled study. Clin Rehabil 2014;28:648-57.

154 Wang X, Che G. Liu L. a short-term high-intensive pattern of preoperative rehabilitation better suits surgical lung cancer patients. Interactive Cardiovascular and Thoracic Surgery 2017;25:i11.
155 Allen S, Brown V, White D. Multi-Modal prehabilitation during neoadjuvant therapy prior to resection for oesophagogastric cancer: a pilot randomised controlled trial. British Journal of Surgery 2019;106:90-1.

156 Lai Y, Wang X, Zhou K, et al. Impact of one-week preoperative physical training on clinical outcomes of surgical lung cancer patients with limited lung function: a randomized trial. Ann Trans/ Med 2019;7) 0(:544. no pagination.

157 Liu Z, Qiu T, Pei L. Two-Week multimodal prehabilitation program improves perioperative functional capability in patients undergoing thoracoscopic lobectomy for lung cancer: a randomized controlled trial. Anesth Analg 2019;23.

158 Vagvolgyi A, Rozgonyi Z, Kerti M, et al. Effectiveness of pulmonary rehabilitation and correlations in between functional parameters, extent of thoracic surgery and severity of post-operative complications: randomized clinical trial. J Thorac Dis 2018;10:3519+ .

159 Minnella EM, Awasthi R, Bousquet-Dion G, et al. Multimodal prehabilitation to enhance functional capacity following radical cystectomy: a randomized controlled trial. Eur Urol Focus 2021;7:132-138.

160 Boden I, Skinner EH, Browning L, et al. Preoperative physiotherapy for the prevention of respiratory complications after upper abdominal surgery: pragmatic, double blinded, multicentre randomised controlled trial. BMJ 2018;360:;5916.

161 Butler GS, Hurley CA, Buchanan KL, et al. Prehospital education: effectiveness with total hip replacement surgery patients. Patient Educ Couns 1996;29:189-97.

162 Cooke M, Walker R, Aitken LM, et al. Pre-Operative self-efficacy education vs. usual care for patients undergoing joint replacement surgery: a pilot randomised controlled trial. Scand J Caring Sci 2016;30:74-82.

163 Eschalier B, Descamps S, Pereira B, et al. Randomized blinded trial of standardized written patient information before total knee arthroplasty. PLoS One 2017;12:e0178358.

164 Giraudet-Le Quintrec J-S, Coste J, Vastel L, et al. Positive effect of patient education for hip surgery: a randomized trial. Clin Orthop Relat Res 2003;414:112-20.

165 Johansson K, Salanterä S, Katajisto J. Empowering orthopaedic patients through preadmission education: results from a clinical study. Patient Educ Couns 2007:66:84-91.

166 Kesänen J, Leino-Kilpi H, Lund T, et al. Increased preoperative knowledge reduces surgery-related anxiety: a randomised clinical trial in 100 spinal stenosis patients. Eur Spine J 2017;26:2520-8.

167 Koyuncu O, Canbulat N, Seyahi A. Does preoperative patient education have an effect on early postoperative pain levels in patients having arthroscopic rotator cuff surgery?, Artroskopik rotator Manset Cerrahisi Oncesi Verilen Hasta Etitiminin Cerrah Sonrasi Erken Donemde Atri Seviyesi Uzerine Etkisi var midir? Turkish, English]. Turkiye Fiziksel Tip ve Rehabilitasyon Dergisi 2013;59:432.

168 Subirana Magdaleno H, Caro Tarragó A, Olona Casas C. Evaluation of the impact of preoperative education in ambulatory laparoscopic cholecystectomy. A prospective, double-blind randomized trial. Cir Esp 2018;96:88-95.

169 McGregor AH, Rylands H, Owen A, et al. Does preoperative hip rehabilitation advice improve recovery and patient satisfaction? J Arthroplasty 2004;19:464-8.

170 Shuldham CM, Fleming S, Goodman H. The impact of preoperative education on recovery following coronary artery bypass surgery. A randomized controlled clinical trial. Eur Heart $J$ 2002;23:666-74.

171 Soeters R, White PB, Murray-Weir M, et al. Preoperative physical therapy education reduces time to meet functional milestones after total joint arthroplasty. Clin Orthop Relat Res 2018;476:40-8.

172 Vukomanović A, Popović Z, Durović A, et al. The effects of short-term preoperative physical therapy and education on early functional recovery of patients younger than 70 undergoing total hip arthroplasty. Vojnosanit Pregl 2008;65:291-7.

173 Watt-Watson J, Stevens B, Costello J, et al. Impact of preoperative education on pain management outcomes after coronary artery bypass graft surgery: a pilot. Can J Nurs Res 2000;31:41-56.

174 Watt-Watson J, Stevens B, Katz J, et al. Impact of preoperative education on pain outcomes after coronary artery bypass graft surgery. Pain 2004;109:73-85.

175 Pazar B, lyigun E. The effects of preoperative education of cardiac patients on haemodynamic parameters, comfort, anxiety and patient-ventilator synchrony: a randomised, controlled trial. Intensive Crit Care Nurs 2020;58:102799.

176 Wilson RA, Watt-Watson J, Hodnett E, et al. A randomized controlled trial of an individualized preoperative education 
intervention for symptom management after total knee arthroplasty. Orthop Nurs 2016:35:20-9.

177 Auer CJ, Laferton JAC, Shedden-Mora MC, et al. Optimizing preoperative expectations leads to a shorter length of hospital stay in CABG patients: further results of the randomized controlled PSY HEART trial. J Psychosom Res 2017;97:82-9.

178 Cohen L, Parker PA, Vence L, et al. Presurgical stress management improves postoperative immune function in men with prostate cancer undergoing radical prostatectomy. Psychosom Med 2011;73:218-25.

179 Furze G, Dumville JC, Miles JNV, et al. "Prehabilitation" prior to CABG surgery improves physical functioning and depression. Int $J$ Cardiol 2009;132:51-8.

180 Goodman H, Parsons A, Davison J, et al. A randomised controlled trial to evaluate a nurse-led programme of support and lifestyle management for patients awaiting cardiac surgery 'Fit for surgery: Fit for life' study. Eur J Cardiovasc Nurs 2008;7:189-95.

181 CTT L, Ong CE, Tang CL. Effects of a psychoeducation intervention on improving outcomes of patients with colorectal cancer: a pilot randomised controlled trial. Psycho-Oncology 2016;25:30-1.

182 Ma YL, Qin LJ, Han ZF. [Relaxation training on stress response to abdominal surgery]. Zhonghua Hu Li Za Zhi 1996;31:377-80.

183 Miró J, Raich RM. Preoperative preparation for surgery: an analysis of the effects of relaxation and information provision. Clinical Psychology \& Psychotherapy 1999;6:202-9.

184 Osinowo HO, Olley BO, Adejumo AO. Evaluation of the effect of cognitive therapy on perioperative anxiety and depression among Nigerian surgical patients. West Afr J Med 2003;22:338-42.

185 Samnani SS, Umer MF, Mehdi SH, et al. Impact of preoperative counselling on early postoperative mobilization and its role in smooth recovery. Int Sch Res Notices 2014;2014:250536-36.

186 Wells JK, Howard GS, Nowlin WF, et al. Presurgical anxiety and postsurgical pain and adjustment: effects of a stress inoculation procedure. J Consult Clin Psychol 1986;54:831-5.

187 Lotzke H, Brisby H, Gutke A, et al. A Person-Centered prehabilitation program based on cognitive-behavioral physical therapy for patients scheduled for lumbar fusion surgery: a randomized controlled trial. Phys Ther 2019;99:1069-88.

188 Medina-Garzón M. Effectiveness of a nursing intervention to diminish preoperative anxiety in patients programmed for knee replacement surgery: preventive controlled and randomized clinical trial. Invest Educ Enferm 2019;37.

189 Marinelli V, Danzi OP, Mazzi MA, et al. Prepare: preoperative anxiety reduction. one-year feasibility RCT on a brief psychological intervention for pancreatic cancer patients prior to major surgery. Front Psychol 2020;11:362.

190 Strøm J, Nielsen CV, Jørgensen LB, et al. A web-based platform to accommodate symptoms of anxiety and depression by featuring social interaction and animated information in patients undergoing lumbar spine fusion: a randomized clinical trial. Spine $J$ 2019;19:827-39.

191 Vlisides PE, Das AR, Thompson AM, et al. Home-Based cognitive prehabilitation in older surgical patients: a feasibility study. $J$ Neurosurg Anesthesiol 2019;31:212-7.

192 Gade H, Hjelmesæth J, Rosenvinge JH, et al. Effectiveness of a cognitive behavioral therapy for dysfunctional eating among patients admitted for bariatric surgery: a randomized controlled trial. J Obes 2014;2014:127936.

193 Andrews K, Bale P, Chu J, et al. A randomized controlled trial to assess the effectiveness of a letter from a consultant surgeon in causing smokers to stop smoking pre-operatively. Public Health 2006:120:356-8.

194 Lee SM, Landry J, Jones PM, et al. The effectiveness of a perioperative smoking cessation program: a randomized clinica trial. Anesth Analg 2013;117:605-13.

195 Myles PS, Leslie K, Angliss M, et al. Effectiveness of bupropion as an aid to stopping smoking before elective surgery: a randomised controlled trial. Anaesthesia 2004:59:1053-8.

196 Nåsell H, Adami J, Samnegård E, et al. Effect of smoking cessation intervention on results of acute fracture surgery: a randomized controlled trial. J Bone Joint Surg Am 2010;92:1335-42.

197 Pei H, Zhang L, Zeng L. Effect of preoperative smoking intervention on postoperative complications of total hip replacement [Chinese]. Chinese Journal of Evidence-Based Medicine 2014;14:399-403.

198 Tonnesen H, Kehlet H. Preoperative alcoholism and postoperative morbidity. Br J Surg 1999;86:869-74.

199 Tønnesen $\mathrm{H}$. Intensive intervention among alcohol patients prior to elective hip Replacement-Complications, Life-Quality, surgical outcome. data reported in Oppedal K, Møller am, Pedersen B, Tønnesen H. Preoperative alcohol cessation prior to elective surgery.
Cochrane Database of Systematic Reviews 2002;2012:Art. No.: CD008343.

200 Anceschi R, Bisi M, Ghidini N, et al. Serenoa repens (Permixon®) reduces intra- and postoperative complications of surgical treatments of benign prostatic hyperplasia. Minerva Urol Nefrol 2010;62:219-23.

201 Chello M, Patti G, Candura D, et al. Effects of atorvastatin on systemic inflammatory response after coronary bypass surgery. Crit Care Med 2006;34:660-7.

202 Marwick TH, Branagan H, Venkatesh B, et al. Use of a nurse-led intervention to optimize beta-blockade for reducing cardiac events after major noncardiac surgery. Am Heart J 2009;157:784-90.

203 Nouri-Majalan N, Ardakani EF, Forouzannia K, et al. Effects of allopurinol and vitamin $E$ on renal function in patients with cardiac coronary artery bypass grafts. Vasc Health Risk Manag 2009;5:489-94

204 Codina S, Coloma A, Sbraga F. SO020NEPHROLOGY intervention in patients awaiting cardiac surgery: a randomised controlled trial. Nephrology Dialysis Transplantation2020;35(Supplement_3.

205 Elrefai M. Value of preoperative diet before sleeve gastrectomy: a prospective randomized study. Obesity Surgery 2019;29:860.

206 Minnella EM, Awasthi R, Loiselle S-E, et al. Effect of exercise and nutrition prehabilitation on functional capacity in esophagogastric cancer surgery: a randomized clinical trial. JAMA Surg 2018;153:1081-9.

207 Karenovics W, Licker M, Ellenberger C, et al. Short-Term preoperative exercise therapy does not improve long-term outcome after lung cancer surgery: a randomized controlled study. Eur $J$ Cardiothorac Surg 2017;52:47-54.

208 Lai Y, Su J, Yang M, et al. [Impact and Effect of Preoperative Shortterm Pulmonary Rehabilitation Training on Lung Cancer Patients with Mild to Moderate Chronic Obstructive Pulmonary Disease: A Randomized Trial]. Zhongguo Fei Ai Za Zhi 2016;19:746-53.

209 Available: http://www.nice.org.uk/guidance/ta182/resources/ guidance-prasugrel-for-the-treatment-of-acute-coronarysyndromes-with-percutaneous-coronary-intervention-pdf

210 Richardson K, Levett DZH, Jack S, et al. Fit for surgery? perspectives on preoperative exercise testing and training. $\mathrm{Br} J$ Anaesth 2017;119:i34-43.

211 Nath B, Li Y, Carroll JE, et al. Alcohol exposure as a risk factor for adverse outcomes in elective surgery. J Gastrointest Surg 2010;14:1732-41.

212 Bamgbade OA, Rutter TW, Nafiu OO, et al. Postoperative complications in obese and nonobese patients. World J Surg 2007:31:556-60. discussion 61.

213 Mondloch MV, Cole DC, Frank JW. Does how you do depend on how you think you'll do? A systematic review of the evidence for a relation between patients' recovery expectations and health outcomes. CMAJ 2001;165:174-9.

214 Armijo-Olivo S, Fuentes J, da Costa BR, et al. Blinding in physica therapy trials and its association with treatment effects: a Metaepidemiological study. Am J Phys Med Rehabil 2017;96:34-44.

215 Short V, Herbert G, Perry R. Chewing gum for postoperative recovery of gastrointestinal function. Cochrane Database Syst Rev 2015;2:Cd006506.

216 López-López JA, Page MJ, Lipsey MW, et al. Dealing with effect size multiplicity in systematic reviews and meta-analyses. Res Synth Methods 2018 doi:10.1002/jrsm.1310

217 Moher D, Liberati A, Tetzlaff J, et al. Preferred reporting items for systematic reviews and meta-analyses: the PRISMA statement. PLoS Med 2009;6:e1000097.

218 van Ginhoven TM, Dik WA, Mitchell JR, et al. Dietary restriction modifies certain aspects of the postoperative acute phase response. J Surg Res 2011;171:582-9.

219 Tenconi S, Galeone C, Fugazzaro S, et al. F-079PERIOPERATIVE and long-term effects of comprehensive pulmonary rehabilitation on exercise capacity, postoperative outcome and quality of life in patients undergoing lung resection: a randomized controlled trial granted by the Ministry of health. Interactive CardioVascular and Thoracic Surgery2017;25.

220 Jarosz A, Szlubowski A, Grochowski Z, et al. P-120 * the evaluation of utility of preoperative Systematized PULMONOLOGICAL physiotherapy among non-small-cell lung cancer patients undergoing anatomical lung resection. Interactive CardioVascular and Thoracic Surgery 2014;18:S32.

221 Subirana Magdaleno H, Caro Tarragó A, Olona Casas C, et al. Evaluation of the impact of preoperative education in ambulatory laparoscopic cholecystectomy. A prospective, double-blind randomized trial. Cir Esp 2018;96:88-95.

222 Tønnesen H, Kehlet $\mathrm{H}$. Preoperative alcoholism and postoperative morbidity. Br J Surg 1999;86:869-74. 